\title{
Dynamic Matching,Two-sided Incomplete Information, and Participation Costs: Existence and Convergence to Perfect Competition
}

\author{
Mark Satterthwaite and Artyom Shneyerov*†
}

December 17, 2004

\begin{abstract}
Consider a decentralized, dynamic market with an infinite horizon and participation costs in which both buyers and sellers have private information concerning their values for the indivisible traded good. Time is discrete, each period has length $\delta$, and each unit of time continuums of new buyers and sellers consider entry. Traders whose expected utility is negative choose not to enter. Within a period each buyer is matched anonymously with a seller and each seller is matched with zero, one, or more buyers. Every seller runs a first price auction with a reservation price and, if trade occurs, both the seller and winning buyer exit the market with their realized utility. Traders who fail to trade continue in the market to be rematched. We characterize the steady-state equilibria that satisfy a subgame perfection criterion. We show that, as $\delta$ converges to zero, equilibrium prices at which trades occur converge to the Walrasian price and the realized allocations converge to the competitive allocation. We also show existence of equilibria for $\delta$ sufficiently small.
\end{abstract}

${ }^{*}$ Kellogg School of Management, Northwestern University and Department of Economics, University of British Columbia respectively.

${ }^{\dagger}$ We owe special thanks to Zvika Neeman who originally devised a proof showing the strict monotonicity of strategies. We also thank Hector Chade, Patrick Francois, Paul Milgrom, Dale Mortensen, Peter Norman, Mike Peters, Jeroen Swinkels, Asher Wolinsky, Jianjun Wu, Okan Yilankaya, and two very perceptive anonymous referees; the participants at the "Electronic Market Design Meeting" (June 2002, Schloss Dagstuhl), the 13th Annual International Conference on Game Theory at Stony Brook, the 2003 NSF Decentralization Conference held at Purdue University, the 2003 General Equilibrium Conference held at Washington University, the 2003 Summer Econometric Society Meeting held at Northwestern University, the 2004 Canadian Economic Theory Conference held in Montreal, and Games 2004 held at Luminy in Marseille; seminar participants at Carnegie-Mellon, Washington University, Northwestern University, University of Michigan, Harvard and MIT, UBC, and Stanford; and the members of the collaborative research group on "Foundations of Electronic Marketplaces" for their constructive comments. Adam Wong provided excellent research assistance. Finally, both of us acknowledge gratefully that this material is based on work supported by the National Science Foundation under Grant IIS-0121541. Artyom Shneyerov also acknowledges support from the Canadian SSHRC Grant 410-2003-1366. 


\section{Introduction}

The frictions of asymmetric information, search costs, time discounting, and strategic behavior interfere with efficient trade. Nevertheless economists have long believed that for private goods' economies the presence of many traders overcomes both these imperfections and results in convergence to perfect competition. Two classes of models demonstrate this. First, double auction models in which traders' costs and values are private exhibit rapid convergence to the competitive price and the efficient allocation within a one-shot centralized market. Second, dynamic matching and bargaining models in which traders' costs and value are common knowledge also converge to the competitive equilibrium. The former models are unrealistic in that they assume traders who fail to trade now can not trade later. Tomorrow (almost) always exists for economic agents. The latter models are unrealistic in assuming traders have no private information. Information about a trader's cost/value (almost) always contains a component that is private to him. This paper's contribution is to formulate a natural model of dynamic matching and bargaining with two-sided incomplete information and show that it converges to the competitive allocation and price as the frictions vanish.

An informal description of our model and result is this. An indivisible good is traded in a market in which time progresses in discrete periods of length $\delta$ and generations of traders overlap. Each unit of time traders who are active in the market incur the frictions of a participation cost $\kappa$ and a discount rate $\beta$. Thus the per period participation cost is $\delta \kappa$, the per period discount factor is $e^{-\beta \delta}$, and the two frictions vanish as the period length becomes short and converges to zero. Each period every active buyer randomly matches with an active seller. Depending on the luck of the draw, a seller may end up being matched with several buyers, a single buyer, or no buyers. Each seller solicits a bid from each buyer with whom she is matched and, if the highest of the bids is satisfactory to her, she sells her single unit of the good and both she and the successful buyer exit the market. A buyer or seller who fails to trade remains in the market, is rematched the next period, and tries again to trade.

Each unit of time a large number of potential sellers (formally, measure 1 of sellers) considers entry into the market along with a large number of potential buyers (formally, measure $a$ of buyers). Each potential seller independently draws a cost $c$ in the unit interval from a distribution $G_{S}$ and each potential buyer draws independently a value $v$ in the unit interval from a distribution $G_{B}$. Individuals' costs and values are private to them. A potential trader only enters the market if, conditional on his private cost or value, his equilibrium expected utility is at least zero. Potential traders whose expected utility is negative elect not to participate.

If trade occurs between a buyer and seller at price $p$, then they exit with their gains from trade, $v-p$ and $p-c$ respectively, less their accumulated participation costs, all discounted back at rate $\beta$ to the time that they entered. If $\delta$ is large (i.e., periods are long), then participation costs accumulate in a short number of periods and a trader who chooses to enter must be confident that he can obtain a profitable trade without much search. If, however, $\delta$ is small, then a trader can wait through many matches looking for a good price with little concern about participation costs and discounting 
offsetting his gains from trade. This option value effect drives the convergence and puts pressure on traders on the opposite side of the market to offer competitive terms. As $\delta$ becomes small the market for each trader becomes, in effect, large. This induces traders to reveal that private information that is necessary for an efficient allocation, just as in the double auction literature.

We characterize steady state equilibria for this market in which each agent maximizes his expected utility going forward. We show that, as the period length goes to zero, all such equilibria converge to the Walrasian price and the competitive allocation. The Walrasian price $p_{W}$ in this market is the solution to the equation

$$
G_{S}\left(p_{W}\right)=a\left(1-G_{B}\left(p_{W}\right)\right),
$$

i.e., it is the price at which the measure of entering sellers with costs less than $p_{W}$ equals the measure of entering buyers with values greater than $p_{W}$. If the market were completely centralized with every active buyer and seller participating in an exchange that cleared each period's bids and offers simultaneously, then $p_{W}$ would be the market clearing price each period. Our precise result is this. Among active traders, let $\bar{c}_{\delta}$ and $\underline{v}_{\delta}$ be the maximal seller's type and minimal buyer's type respectively and let $\left[\underline{p} \underline{p}_{\delta}, \bar{p}_{\delta}\right]$ be the range of prices at which trades occur. Also let $\underline{c}_{\delta}$ be the smallest bid acceptable to any active seller. As $\delta \rightarrow 0$, then $\underline{c}_{\delta}, \bar{c}_{\delta}, \underline{v}_{\delta}, \underline{p}_{\delta}$, and $\bar{p}_{\delta}$ all converge to the same limit $p$. In the steady-state, the only way for the market to clear is for this limit $p$ to be equal to the competitive price $p_{W}$. That the resulting allocations give traders the expected utility they would realize in a perfectly competitive market follows. Finally, we show that these equilibria exist if $\delta$ is sufficiently small.

A substantial literature exists that investigates the non-cooperative foundations of perfect competition using dynamic matching and bargaining games. ${ }^{1}$ Most of the work of which we are aware has assumed complete information in the sense that each participant knows every other participant's values (or costs) for the traded good. The books of Osborne and Rubinstein (1990) and Gale (2000) contain excellent discussions of both their own and others' contributions to this literature. Papers that have been particularly influential include Mortensen (1982), Rubinstein and Wolinsky (1985, 1990), Gale $(1986,1987)$ and Mortensen and Wright (2002). Of these, our paper is most closely related to the models and results of Gale (1987) and Mortensen and Wright (2002). The two main differences between their work and ours are that (i) when two traders meet they reciprocally observe the other's cost/value rather than remaining uninformed and (ii) the terms of trade are determined as the outcome of a full information bargaining game rather than an auction. The first difference - full versus incomplete informationis fundamental, for the purpose of our paper is to determine if a decentralized market can elicit private valuation information at the same time it uses that information to assign the available supply efficiently. The second difference is natural given our focus on incomplete information.

\footnotetext{
${ }^{1}$ There is a related literature that we do not discuss here concerning is the micro-structure of intermediaries in markets, e.g., Spulber (1999) and Rust and Hall (2002). These models allow entry of an intermediary who posts fixed ask and offer prices and is assumed to be large enough to honor any size buy or sell order without exhausting its inventory or financial resources.
} 
The most important dynamic bargaining and matching models that incorporate incomplete information are Wolinsky (1988), De Fraja and Sákovics (2001), and Serrano (2002). ${ }^{23}$ To understand how our paper relates to these papers, consider the following problem as the baseline. Each unit of time measure 1 sellers and measure $a$ buyers enter the market, each of whom has a private cost/value for a single unit of the homogeneous good. The sellers' units of supply need to be reallocated to those traders who most highly value them. Whatever mechanism that is employed must induce the traders to reveal sufficient information about their costs/valuations in order to carry out the reallocation. The static double auction literature- perhaps most notably Satterthwaite and Williams (1989) and Rustichini, Satterthwaite and Williams (1994) in the independent private values case - shows that even moderately-sized centralized double auction held once per unit time solves this problem essentially perfectly by closely approximating the Walrasian price and then using that price to mediate trade. ${ }^{4}$

Given this definition of the problem, the reason why Wolinsky (1988), De Fraja and Sákovics (2001), and Serrano (2002) do not obtain competitive outcomes as the frictions in their models vanish is clear: the problems their models address are different and, as their results establish, not intrinsically perfectly competitive even when the market becomes almost frictionless. Wolinsky's model relaxes the homogeneous good assumption and does not fully analyze the effects of entry/exit dynamics. De Fraja and Sákovics' model's entry/exit dynamics do not specify fixed measures of buyers and sellers entering the market each unit of time and therefore have no market clearing force moving the market towards a supply-demand equilibrium. Serrano's model embeds a discrete-price double auction mechanism in a dynamic matching framework. In his model, however, there are no entering cohorts of traders. Consequently, the option-value effects become progressively smaller as the most avid buyers and sellers leave the market through trading. Serrano finds that "equilibria with Walrasian and non-Walrasian features persist." These papers complement our investigation of sufficient conditions for convergence because, as we discuss in the conclusions, they implicitly identify necessary conditions for convergence and bring closer the goal of a full characterization of convergent matching and bargaining models.

The next section formally states the model and our main result establishing that the

\footnotetext{
${ }^{2}$ Butters in an unfinished manuscript (circa 1979) that was well before its time considers convergence in a dynamic matching and bargaining problem. The main differences between our model and his are (i) he assumes an exogenous exit rate instead of a participation cost, (ii) traders who have zero probability of trade participate in the market until they exit stochastically due to the exogenous exit rate, and (iii) the matching is one-to-one and the matching probabilities do not depend on the ratio of buyers and sellers in the market. We thank Asher Wolinsky for bringing Butters' manuscript to our attention after we had completed an earlier version of this paper.

${ }^{3}$ In a working paper Satterthwaite and Shneyerov (2003) show convergence in a dynamic matching and bargaining model that has no participation costs, but instead has the alternative friction of a fixed, exogenous, per unit time rate of exit among active traders. Market clearing is more subtle in this alternative model and as a consequence equilbria in it have a different structure than the equilibria we study in this paper.

${ }^{4}$ Another example of a centralized trading institution is the system of simultaneous ascending-price auctions, studied in Peters and Severinov (2002). They also find robust convergence to the competitive outcome.
} 
Walrasian price robustly emerges as the market becomes increasing frictionless. Section 3 derives basic properties of equilibria and Section 4 proves our main result. Section 5 shows existence of equilibrium if $\delta$ is sufficiently small by identifying a class of full-trade equilibria and proving that, for sufficiently small $\delta$, such equilibria exist and are unique. Section 7 concludes with a discussion of some important possible extensions that merit future attention.

\section{Model and theorem}

We study the steady state of a market with two-sided incomplete information and an infinite horizon. In it heterogeneous buyers and sellers meet once per period $(t=$ $\ldots,-1,0,1, \ldots)$ and trade an indivisible, homogeneous good. Every seller is endowed with one unit of the traded good and her cost is $c \in[0,1]$. This cost is private information to her; to other traders it is an independent random variable with distribution $G_{S}$ and density $g_{S}$. Similarly, every buyer seeks to purchase one unit of the good and his value is $v \in[0,1]$. This value is private; to others it is an independent random variable with distribution $G_{B}$ and density $g_{B}$. Our model is therefore the standard independent private values model. We assume that the two densities are bounded away from zero: a $\underline{g}>0$ exists such that, for all $c, v \in[0,1], g_{S}(c)>\underline{g}$ and $g_{B}(v)>\underline{g}$.

The strategy of a seller, $S:[0,1] \rightarrow R \cup\{\mathcal{N}\}$, maps her cost $c$ into either a decision $\mathcal{N}$ not to enter or a minimal bid that she is willing to accept. Similarly the strategy of a seller, $B:[0,1] \rightarrow R \cup\{\mathcal{N}\}$, maps his value $v$ into either a decision $\mathcal{N}$ not to enter or the bid that he places when he is matched with a seller.

The length of each period is $\delta$. Each unit of time measure 1 of potential sellers and measure $a$ of potential buyers consider entry where $a>0$. Potential traders receive utility of 0 if they choose not to enter. This means that each period measure $\delta$ of potential sellers and measure $\delta a$ of potential buyers consider entry. A period consists of five steps:

1. Entry occurs. A type $v$ potential buyer becomes active only if $B(v) \neq \mathcal{N}$ and a type $c$ potential seller becomes active only if $S(c) \neq \mathcal{N}$.

2. Every active seller and buyer incurs participation cost $\delta \kappa$.

3. Every buyer is matched with one seller. The probability $\pi_{k}$ that a seller is matched with $k \in\{0,1,2, \ldots\}$ buyers is Poisson:

$$
\pi_{k}(\zeta)=\frac{\zeta^{k}}{k ! e^{\zeta}}
$$

where $\zeta$ is the endogenous ratio of active buyers to active sellers. ${ }^{5}$ Consequently a seller may end up being matched with zero buyers, one buyer, two buyers, etc.

\footnotetext{
${ }^{5}$ In a market with $M$ sellers and $\zeta M$ buyers, the probability that a seller is matched with $k$ buyers is $\pi_{k}^{M}=\left(\begin{array}{c}\zeta M \\ k\end{array}\right)\left(\frac{1}{M}\right)^{k}\left(1-\frac{1}{M}\right)^{\zeta M-k}$. Poisson's theorem (see, for example, Shiryaev, 1995) shows that $\lim _{M \rightarrow \infty} \pi_{k}^{M}=\pi_{k}$.
} 
4. Each buyer simultaneously announces a bid $B(v)$ to the seller with whom he is matched. We assume that, at the time he submits his bid, each buyer only knows the endogenous steady-state probability distribution of how many buyers with whom he is competing. After receiving the bids, the seller either accepts or rejects the highest bid. Denote by $S(c)$ the minimal bid acceptable to a type $c$ seller. If two or more buyers tie with the highest bid, then the seller uses a fair lottery to choose between them. If a type $v$ buyer trades in period $t$, then he leaves the market with utility $v-B(v)$. If a type $c$ seller trades at price $p$, then she leaves the market with utility $p-c$ where $p$ is the bid she accepts.

5. All remaining traders carry over to the next period.

Traders discount their expected utility at the rate $\beta \geq 0$ per unit time; $e^{-\beta \delta}$ is therefore the factor by which each trader discounts his utility per period of time.

To formalize the fact that the distribution of trader types within the market's steady state is endogenous, let $T_{S}$ be the measure of active sellers in the market at the beginning of each period, $T_{B}$ be the measure of active buyers, $F_{S}$ be the distribution of active seller types, and $F_{B}$ be the distribution of active buyer types. The corresponding densities are $f_{S}$ and $f_{B}$ and, establishing useful notation, the right-hand distributions are $\bar{F}_{S} \equiv 1-F_{S}$ and $\bar{F}_{B} \equiv 1-F_{B}$. The ratio $\zeta$ is therefore equal to $T_{B} / T_{S}$.

By a steady state equilibrium we mean one in which every seller in every period plays a symmetric, time invariant strategy $S(\cdot)$, every buyer plays a symmetric, time invariant strategy $B(\cdot)$, and both these strategies are always optimal. Let $W_{S}(c)$ and $W_{B}(v)$ be the sellers and buyers' interim utilities for sellers of type $c$ and the buyers of type $v$ respectively, i.e, they are beginning-of-period, steady-state, equilibrium net payoffs conditional on their types. Given the friction $\delta$, a market equilibrium $M_{\delta}$ consists of strategies $\{S, B\}$, traders' masses $\left\{T_{S}, T_{B}\right\}$, and distributions $\left\{F_{S}, F_{B}\right\}$ such that (i) $\{S, B\},\left\{T_{S}, T_{B}\right\}$, and $\left\{F_{S}, F_{B}\right\}$ generate $\left\{T_{S}, T_{B}\right\}$ and $\left\{F_{S}, F_{B}\right\}$ as their steady state and (ii) no type of trader can increase his or her expected utility (including the continuation payoff from matching in future periods if trade fails) by a unilateral deviation from the strategies $\{S, B\}$, and (iii) equilibrium strategies $\{S, B\}$, masses $\left\{T_{S}, T_{B}\right\}$, and distributions $\left\{F_{S}, F_{B}\right\}$ are common knowledge among all active and potential traders. The equilibrium is required assumed to be subgame perfect.

Three points need emphasis concerning this definition. First, since within a given match buyers announce their bids simultaneously and only then does the seller decide to accept or reject the highest of the bids, subgame perfection implies that a seller whose highest received bid is above her total dynamic opportunity cost of $c+e^{-\beta \delta} W_{S}(c)$ accepts that bid. In other words, a seller's strategy is her full dynamic opportunity cost,

$$
S(c)=c+e^{-\beta \delta} W_{S}(c),
$$

and is independent of the number of buyers who are bidding, i.e., $S(c)$ is her reservation price. Second, beliefs are simple to handle because our twin assumptions that there are continuums of traders and that all matching is anonymous and independent imply that off-the-equilibrium path actions do not cause any inference ambiguities. Third, every 
trader who enters eventually succeeds in trading, i.e., exit only takes place as a result of trade. This fact, as the proof of Proposition 8 below demonstrates, insures that, as the period length becomes short, the prices at which trades occur in the steady state must converge to the Walrasian price $p_{W}$.

Define $\bar{c}$ and $\underline{v}$ to be the maximal seller and minimal buyer types that choose to enter. These are the marginal participation types. Define $\underline{c}, \underline{p}$, and $\bar{p}$ to be the minimal reservation price, minimal bid, and maximal bid that are made in equilibrium. Formally, let $A_{S} \subset[0,1]$ and $A_{B} \subset[0,1]$ be the sets of active traders' types; then

$$
\begin{aligned}
\bar{c} & \equiv \sup \left\{c \mid c \in A_{S}\right\}, \\
\underline{v} & \equiv \inf \left\{v \mid v \in A_{B}\right\}, \\
\underline{c} & =\inf \left\{S(c) \mid c \in A_{S}\right\}, \\
\underline{p} & =\inf \left\{B(v) \mid v \in A_{B}\right\}, \\
\bar{p} & =\sup \left\{B(v) \mid v \in A_{B}\right\} .
\end{aligned}
$$

Given an equilibrium $M_{\delta}$, we index with $\delta$ both its components $S_{\delta}, B_{\delta}, F_{S \delta}, F_{B \delta}, T_{S \delta}$, $T_{B \delta}$, and $\zeta_{\delta}$ and its descriptors $\bar{c}_{\delta}, \underline{v}_{\delta}, \underline{c}_{\delta}, \underline{p}_{\delta}$, and $\bar{p}_{\delta}$. With this notation we can state our convergence result:

Theorem 1 Let $M_{\delta}$ be a market equilibrium in which trade occurs, let $\left\{\bar{c}_{\delta}, \underline{v}_{\delta}, \underline{c}_{\delta}, \underline{p}_{\delta}, \bar{p}_{\delta}\right\}$ be the descriptors of its equilibrium strategies, and let $W_{S \delta}(c)$ and $W_{B \delta}(v)$ be traders' interim expected utilities. Then

$$
\lim _{\delta \rightarrow 0} \underline{c}_{\delta}=\lim _{\delta \rightarrow 0} \bar{c}_{\delta}=\lim _{\delta \rightarrow 0} \underline{v}_{\delta}=\lim \underline{p}_{\delta}=\lim _{\delta \rightarrow 0} \bar{p}_{\delta}=p_{W} .
$$

In addition, each trader's interim expected utility converges to the utility he would realize if the market were perfectly competitive:

$$
\lim _{\delta \rightarrow 0} W_{S \delta}(c)=\max \left[0, p_{W}-c\right]
$$

and

$$
\lim _{\delta \rightarrow 0} W_{B \delta}(v)=\max \left[0, v-p_{W}\right] .
$$

Sections 3 and 4 below prove this. In section 5 we prove existence for sufficiently small $\delta$ :

Theorem 2 For all sufficiently small $\delta>0$, an equilibrium $M_{\delta}$ exists in which positive trade occurs.

The purpose of the restriction to equilibria in which positive trade occurs is to rule out the trivial, no-trade equilibrium in which neither buyers nor sellers ever enter the market.

The intuition for our convergence result can be understood through the following logic. In a match in which a buyer is bidding for an object, the "type" that is relevant is not his static type $v$ but, rather, his full dynamic opportunity value

$$
I_{B \delta}(v)=v-e^{-\beta \delta} W_{B \delta}(v) .
$$


Similarly the cost that is relevant to a type $c$ seller is not $c$, but is her full dynamic opportunity cost

$$
I_{S \delta}(c)=c+e^{-\beta \delta} W_{S \delta}(c) .
$$

When the buyers bid in the auction, they act as if their types were drawn from the density $h_{B \delta}(\cdot)$ of $I_{B \delta}(v)$ and the sellers' types were drawn from the density $h_{S \delta}(\cdot)$ of $I_{S \delta}(c)$. Since $\lim _{\delta \rightarrow 0} W_{S \delta}(c)=\max \left[0, p_{W}-c\right]$ and $\lim _{\delta \rightarrow 0} W_{B \delta}(v)=\max \left[0, v-p_{W}\right]$, the theorem indicates that, as the time period length $\delta \rightarrow 0$, the distributions of the "dynamic types" $I_{B \delta}(v)$ and $I_{S \delta}(c)$ become degenerate: both become concentrated around the same point, namely $p_{W}$. Viewed this way, as $\delta \rightarrow 0$, the dynamic matching and bargaining market in equilibrium progressively exhibits less and less heterogeneity among buyers and sellers until there is none - the relevant traders' types converge to $p_{W}$ and the incomplete information vanishes. The underlying driver causing the heterogeneity to vanish as $\delta \rightarrow 0$ is the option value that each trader's optimal search generates.

It is important to point out that in the full information matching and bargaining models of Gale (1987) and Mortensen and Wright (2002) this same mechanism drives convergence: the option value that optimal search creates causes the distributions of buyers and sellers' full opportunity costs to become degenerate as the friction goes to zero. Once this understood, our result that incomplete information does not disrupt convergence is natural. The parallel, however, is not perfect because the proof of convergence in the complete information models depends on the details of the complete information bargaining used.

Figure 1 is a table of graphs illustrating the general character of these equilibria and the manner in which they converge. These computed examples assume that the primitive distributions $G_{S}$ and $G_{B}$ are uniform on [0,1], equal masses of buyers and sellers consider entry each unit of time (i.e., $a=1$ ), and the participation cost is $\kappa=0.01 .^{6}$ The left column shows an equilibrium for $\delta=0.4$ while the right column shows an equilibrium for $\delta=0.2$. Traders' costs and values, $c$ and $v$, are on each graph's abscissa. The top graph in each row shows strategies: sellers' strategies $S(c)$ are to the left and above the diagonal while buyers' strategies $B(v)$ are to the right and below diagonal. Because masses of entering traders are equal and their cost/value distributions are uniform, the Walrasian price is 0.5 ; this is the horizontal line cutting the center of the graph. Observe also that $B(v)$ and $S(c)$ are not defined for non-entering types. The middle graph in each column shows the endogenous densities, $f_{S}$ and $f_{B}$, of active traders in the equilibrium. The density $f_{S}$ for active sellers is on the left and the density for active buyers on the right. Note that to the right of $\bar{c}$ the density $f_{S}$ is zero because sellers with $c>\bar{c}$ choose not to enter. Similarly, to the left of $\underline{v}$ the density $f_{B}$ is zero. The bottom graph in each column shows the equilibrium densities $h_{S}$ and $h_{B}$ of the full opportunity costs and values.

Comparison of the strategies in the top two graphs illustrates the convergence of all the descriptors $\left\{\bar{c}_{\delta}, \underline{v}_{\delta}, \underline{c}_{\delta}, \underline{p}_{\delta}, \bar{p}_{\delta}\right\}$ toward $p_{W}$ as $\delta$ decreases from 0.4 to 0.2. Comparison

\footnotetext{
${ }^{6}$ In computing these equilibria we fit sixth degree Chebyshev polynomials to a system of differential equations and boundary conditions that correspond to the first order conditions and steady state conditions of the problem. The Mathematica program that was used is available upon request.
} 

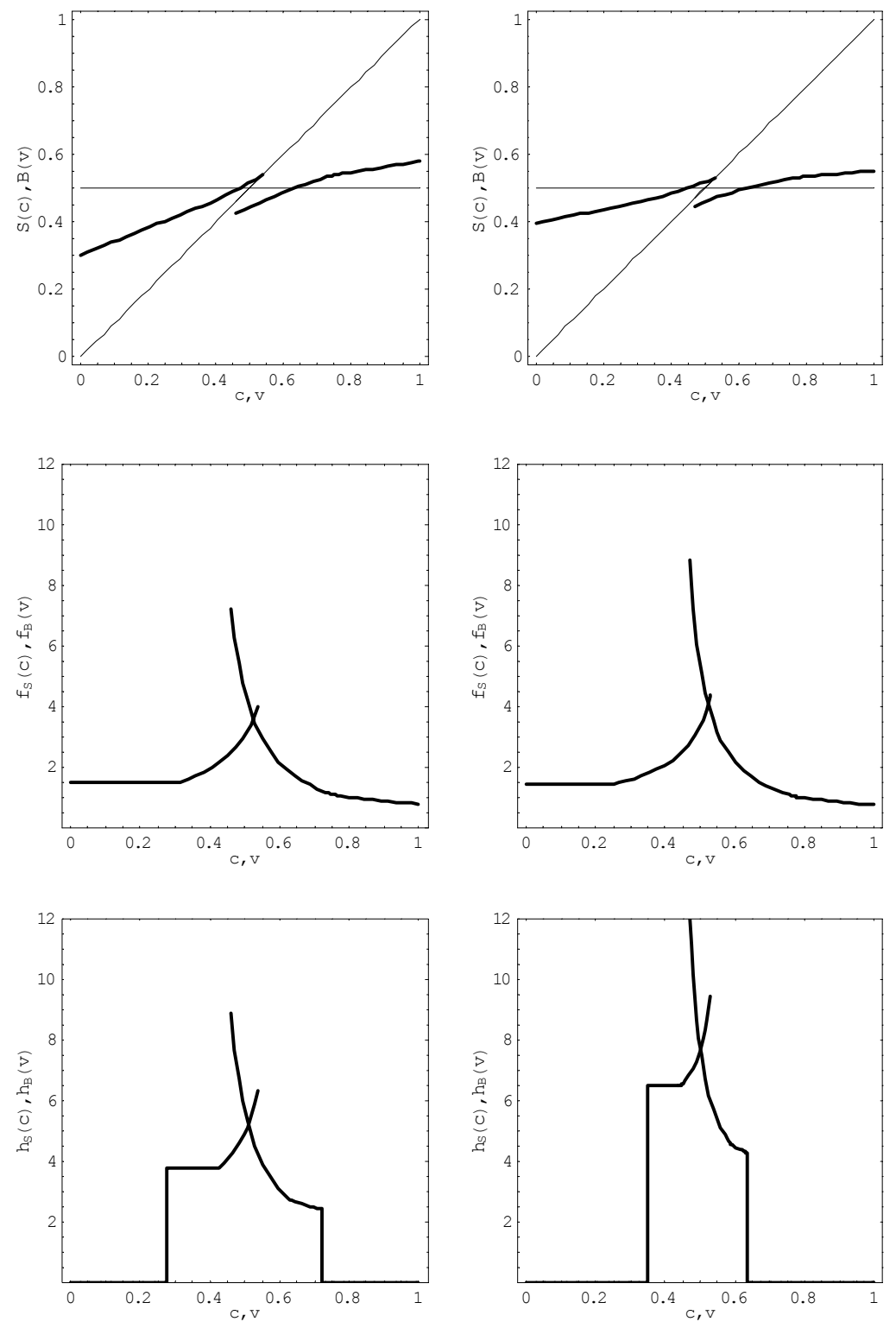

Figure 1: Equilibrium strategies and associated steady state densities. The left column of graphs is for $\delta=0.4$ and the right column is for $\delta=0.2$. The top row shows buyer and seller strategies $S$ and $B$. The middle row graphs the densities $f_{S}$ and $f_{B}$ of the traders' types and the bottom row graphs the densities $h_{S}$ and $h_{B}$ of the traders' full opportunity costs and values. 
of the two bottom graphs shows the reduction of incomplete information as $\delta$ decreases. In these equilibria the presence of incomplete information reduces the efficiency of the equilibria as a result of each buyer shading his bid below his full dynamic value $I_{B \delta}(v)$. This loss, however, is less in the right graph with its lower value of $\delta$ because of the greater concentration of the two densities in the neighborhood of 0.5 .

One final comment concerning the model and theorems is important. In setting up the model we assume that traders use symmetric pure strategies. We do this for simplicity of exposition. At a cost in notation we could define trader-specific and mixed strategies and then prove that they in fact must be symmetric and (essentially) pure because of the anonymous nature of matching and the strict monotonicity of strategies. To see this, first consider the implication of anonymous matching for buyers. Even if different traders follow distinct strategies, every buyer would still draw his opponents from the same population of active traders. ${ }^{7}$ Therefore, for a given value $v$, every buyer will have the identical best response correspondence. Second, we show below that every selection from this correspondence is strictly increasing; consequently, the best response is pure apart from a measure zero set of values where jumps occur. These jump points are the only points where mixing can occur, but because their measure is zero, the mixing has no consequence for the maximization problems of the other traders.

\section{Basic properties of equilibria}

In this section we derive formulas for probabilities of trade and establish the strict monotonicity of strategies. These facts are inputs into the next two sections' proofs. We separate them out because they apply for all $\delta>0$.

\subsection{Discounted ultimate probability of trade and participation cost}

An essential construct for the analysis of our model is the discounted ultimate probability of trade. It allows a trader's expected gains from participating in the market to be written as simply as possible. Let, in the steady state, $\rho_{S}(\lambda)$ be the probability that in a given period a seller who chooses reservation price $\lambda$ trades and, similarly, let $\rho_{B}(\lambda)$ be the probability that a buyer who bids $\lambda$ trades. Also, let $\bar{\rho}_{S}(\lambda)=1-\rho_{S}(\lambda)$ and $\bar{\rho}_{B}(\lambda)=1-\rho_{B}(\lambda)$.

Define recursively $P_{B}(\lambda)$ to be a buyer's discounted ultimate probability of trade if he bids $\lambda$ :

$$
P_{B}(\lambda)=\rho_{B}(\lambda)+\bar{\rho}_{B}(\lambda) e^{-\beta \delta} P_{B}(\lambda) .
$$

Therefore

$$
P_{B}(\lambda)=\frac{\rho_{B}(\lambda)}{1-e^{-\beta \delta}+e^{-\beta \delta} \rho_{B}(\lambda)} .
$$

Observe that the formula incorporates traders' time discounting into the probability calculation. The parallel recursion for sellers implies that

\footnotetext{
${ }^{7}$ This is strictly true because we assume a continuum of traders.
} 


$$
P_{S}(\lambda)=\frac{\rho_{S}(\lambda)}{1-e^{-\beta \delta}+e^{-\beta \delta} \rho_{S}(\lambda)} .
$$

This construct is useful within a steady state equilibrium because it converts the buyer's dynamic decision problem into a static decision problem. Specifically, if successfully trading gives the buyer an expected gain $U$, then his discounted expected utility $W^{B}$ from following the stationary strategy of bidding $\lambda$ is

$$
W^{B}(\lambda, U)=\rho_{B}(\lambda) U-\kappa \delta+\bar{\rho}_{B}(\lambda) e^{-\beta \delta} W^{B}(\lambda, U) .
$$

Solving this recursion gives the explicit formula:

$$
W^{B}(\lambda, U)=P_{B}(\lambda) U-K_{B}(\lambda),
$$

where

$$
\begin{aligned}
K_{B}(\lambda) & =\frac{\kappa \delta}{1-e^{-\beta \delta}+e^{-\beta \delta} \rho_{B}(\lambda)} \\
& =\kappa \delta \frac{P_{B}(\lambda)}{\rho_{B}(\lambda)}
\end{aligned}
$$

is the discounted participation cost over the buyer's lifetime in the market. Similarly,

$$
W^{S}(\lambda, U)=P_{S}(\lambda) U-K_{S}(\lambda)
$$

where

$$
K_{S}(\lambda)=\frac{\kappa \delta}{1-e^{-\beta \delta}+e^{-\beta \delta} \rho_{S}(\lambda)} .
$$

In accord with our convention for non-entering types, we assume that

$$
\rho_{B}(\mathcal{N})=\rho_{S}(\mathcal{N})=K_{B}(\mathcal{N})=K_{S}(\mathcal{N})=0 .
$$

In section 3.3 we derive explicit formulas for $\rho_{B}(\cdot)$ and $\rho_{S}(\cdot)$

\subsection{Strategies are strictly increasing}

This subsection demonstrates the most basic property that our equilibria satisfy: strategies are strictly increasing. We need the following preliminary result.

Lemma 3 In equilibrium, $P_{B}[B(\cdot)]$ is non-decreasing and $P_{S}[S(\cdot)]$ is non-increasing over $[0,1]$. The buyers for whom $v>\underline{v}$ elect to enter, while the buyers for whom $v<\underline{v}$ do not:

$$
(\underline{v}, 1] \subset A_{B}, \quad[0, \bar{v}) \subset \bar{A}_{B} .
$$

The type $\underline{v}$ is indifferent between entering and not entering. Similarly,

$$
[0, \bar{c}) \subset A_{S}, \quad(\bar{c}, 1] \subset \bar{A}_{S}
$$

and the type $\bar{c}$ is indifferent between entering or not. 
Proof. $W_{B}(v)=\sup _{\lambda \in R \cup\{\mathcal{N}\}}(v-\lambda) P_{B}(\lambda)-K_{B}(\lambda)=(v-B(v)) P_{B}(B(v))-$ $K_{B}(B(v))$ is the upper envelope of a set of affine functions. It follows by the envelope theorem that $W_{B}(\cdot)$ is a continuous, increasing and convex function. Because $W_{B}$ is continuous, the definition of $\underline{v}=\inf \left\{v: v \in A_{B}\right\}$ implies that (i) $W_{B}(\underline{v})=0$ and $\underline{v}$ is indifferent between entering or not, and (ii) the types $v<\underline{v}$ prefer not to enter. Further, convexity implies that $W_{B}^{\prime}(\cdot)$ is non-decreasing. By the envelope theorem $W_{B}^{\prime}(\cdot)=P_{B}[B(\cdot)] ; P_{B}[B(\cdot)]$ is therefore non-decreasing at all differentiable points. Milgrom and Segal's (2002) theorem 1 implies that at non-differentiable points $v^{\prime} \in[0,1]$

$$
\lim _{v \rightarrow v^{\prime}-} W_{B}^{\prime}(v) \leq P_{B}\left(B\left(v^{\prime}\right)\right) \leq \lim _{v \rightarrow v^{\prime}+} W_{B}^{\prime}(v) .
$$

Thus $P_{B}[B(\cdot)]$ is everywhere non-decreasing for any best-response $B$. Further, Milgrom and Segal's theorem 2 implies that

$$
W_{B}(v)=W_{B}(\underline{v})+\int_{\underline{v}}^{v} P_{B}[B(x)] d x \quad \text { for } v \geq \underline{v} .
$$

Since $\underline{v}$ is indifferent between entering or not, we can choose a best-response $\widetilde{B}$ in which $\underline{v}$ is active, while $\widetilde{B}(v)=B(v)$ for $v \neq \underline{v}$. $\widetilde{B}$ may different from $B$ at $v=\underline{v}$, since in $\widetilde{B}$, the type $\underline{v}$ is active, while in $B$ it may not be. Importantly, the function $W_{B}(\cdot)$ is the same for both $B$ and $\widetilde{B}$, since by Milgrom and Segal's theorem 2, the envelope condition (12) holds for any selection from the best-response correspondence. Now $P_{B}[\widetilde{B}(\underline{v})]>0$ since otherwise the active buyer $\underline{v}$ would not be able to recover his positive participation cost. Since $P_{B}[\widetilde{B}(\cdot)]$ is non-decreasing, $P_{B}[\widetilde{B}(v)]>0$ for all $v \geq \underline{v}$, and the envelope condition (12) then implies that the buyers for whom $v>\underline{v}$ elect enter. The argument for the sellers is parallel and is omitted.

For notational simplicity we assume from now on that $\underline{v}$ and $\bar{c}$ enter. Since $\{\underline{v}\}$ and $\{\bar{c}\}$ have measure 0 , all our results will hold in substance under the alternative assumption that $\underline{v}$ does not enter.

Lemma $4 B$ is strictly increasing on $[\underline{v}, 1]$.

Proof. Pick any $v, v^{\prime} \in[\underline{v}, 1]$ such that $v<v^{\prime}$. Since $P_{B}[B(\cdot)]$ is non-decreasing, $P_{B}[B(v)] \leq P_{B}\left[B\left(v^{\prime}\right)\right]$ necessarily. We first show that $B$ is non-decreasing on $[\underline{v}, 1]$. Suppose, to the contrary, that $B(v)>B\left(v^{\prime}\right)$. The auction rules imply that $P_{B}(\cdot)$ is nondecreasing; therefore $P_{B}[B(v)] \geq P_{B}\left[B\left(v^{\prime}\right)\right]$. Consequently $P_{B}[B(v)]=P_{B}\left[B\left(v^{\prime}\right)\right]>$ 0 . But this gives $v$ incentive to lower his bid to $B\left(v^{\prime}\right)$, since by doing so he will buy with the same positive probability but pay a lower price. This contradicts $B$ being an optimal strategy and establishes that $B$ is non-decreasing. If $B\left(v^{\prime}\right)=B(v)(=\lambda)$ because $B$ is not strictly increasing, then any buyer with $v^{\prime \prime} \in\left(v, v^{\prime}\right)$ will raise his bid infinitesimally from $\lambda$ to $\lambda^{\prime}>\lambda$ to avoid the rationing that results from a tie. This proves that $B$ is strictly increasing on $[\underline{v}, 1] .^{8}$

\footnotetext{
${ }^{8}$ Alternatively, one can use Theorem 2.2 in Satterthwaite and Williams (1989) with only trivial adaptations.
} 
Lemma $5 S$ is continuous and strictly increasing on $[0, \bar{c}]$.

Proof. Any active seller will accept the highest bid she receives, provided it is above her total opportunity cost:

$$
S(c)=c+e^{-\beta \delta} W_{S}(c)
$$

Milgrom and Segal's Theorem 2 implies that $W_{S}(\cdot)$ is continuous and can be written, for any active seller type $c$ as

$$
\begin{aligned}
W_{S}(c) & =W_{S}(\bar{c})+\int_{c}^{\bar{c}} P_{S}(S(x)) d x \\
& =\int_{c}^{\bar{c}} P_{S}(S(x)) d x
\end{aligned}
$$

where the second line follows from the definition of $\bar{c}$ and the continuity of $W_{S}(\cdot)$. Combining (13) and (14) we see that

$$
S(c)=c+e^{-\beta \delta} \int_{c}^{\bar{c}} P_{S}(S(x)) d x
$$

for all sellers that are active.

When combined with equation (13), it also implies that $S(\cdot)$ is continuous. Therefore, for almost all active sellers $c \in[0, \bar{c}]$,

$$
S^{\prime}(c)=1-e^{-\beta \delta} P_{S}[S(c)]>0
$$

because $W_{S}^{\prime}(c)=-P_{S}[S(c)]$. Since $S(\cdot)$ is continuous, this is sufficient to establish that $S(\cdot)$ is strictly increasing for all active sellers $c \in[0, \bar{c}]$.

Lemma $6 \underline{c}<B(\underline{v})<\underline{v}, S(\bar{c})=\bar{c}<\bar{p}$, and $B(\underline{v}) \leq \bar{c}$.

Proof. Given that $S$ is strictly increasing, $S(0)=\underline{c}$ is the lowest reservation price any seller ever has. A buyer with valuation $v<\underline{c}$ does not enter the market since he can only hope to trade by submitting a bid at or above $\underline{c}$, i.e. above his valuation. In equilibrium, any buyer who enters the market must submit a bid below his valuation and above $\underline{c}$, since otherwise he is unable to recover a positive participation cost. It follows that $\underline{c}<B(\underline{v})<\underline{v}$. Similarly, a seller who is only willing to accept a bid at or above $\bar{p}$ never enters the market, since she is unable to recover her participation cost. This implies $S(\bar{c})<\bar{p}$. Any active seller has acceptance strategy given by (13), so in particular $S(\bar{c})=\bar{c}$.

Finally, suppose that $B(\underline{v})>\bar{c}$. Then the buyer for whom $v=\underline{v}$ bids more than necessary to win the object: he can only be successful if there are no rival buyers, and when this is the case, bidding $\bar{c}$ is sufficient to secure acceptance of the bid by the seller

All these findings are summarized as follows. 
Proposition 7 Suppose that $\{B, S\}$ is a stationary equilibrium. Then, over $[\underline{v}, 1]$ and $[0, \bar{c}], B$ and $S$ are strictly increasing, $S$ is continuous and, almost everywhere on $[0, \bar{c}]$, has derivative

$$
S^{\prime}(c)=1-e^{-\beta \delta} P_{S}[S(c)]
$$

Finally, $B$ and $S$ have the properties that $\underline{c}<\underline{p}<\underline{v}, S(\bar{c})=\bar{c}<\bar{p}$ and $\underline{p} \leq \bar{c}$.

The strict monotonicity of $B$ on $[\underline{v}, 1]$ and $S$ on $[0, \bar{c}]$ allows us to define $V$ and $C$, their inverses over $[B(\underline{v}), B(1)]$ and $[S(0), S(\bar{c})]$ :

$$
\begin{aligned}
& V(\lambda)=\inf \{v \in[0,1]: B(v)>\lambda\}, \\
& C(\lambda)=\inf \{c \in[0,1]: S(c)>\lambda\} .
\end{aligned}
$$

\subsection{Explicit formulas for the probabilities of trading}

Focus on a seller of type $c$ who in equilibrium has a positive probability of trade. In a given period she is matched with zero buyers with probability $\pi_{0}$ and with one or more buyers with probability $\bar{\pi}_{0}=1-\pi_{0}$. Suppose she is matched and $v^{*}$ is the highest type buyer with whom she is matched. Since by Proposition 7 each buyer's bid function $B(\cdot)$ is increasing, she accepts his bid if and only if $B\left(v^{*}\right) \geq \lambda$ where $\lambda$ is her reservation price. The distribution from which $v^{*}$ is drawn is $F_{B}^{*}(\cdot)$ : for $v \in[\underline{v}, 1]$,

$$
F_{B}^{*}(v)=\frac{1}{\bar{\pi}_{0}(\zeta)} \sum_{i=1}^{\infty} \pi_{i}(\zeta)\left[F_{B}(v)\right]^{i}
$$

where $F_{B}(\cdot)$ is the steady state distribution of buyer types and $\left\{\pi_{0}, \pi_{1}, \pi_{2}, \ldots\right\}$ are the probabilities with which each seller is matched with zero, one, two, or more buyers. Note that this distribution is conditional on the seller being matched. Thus if a seller has reservation price $\lambda$, her probability of trading in a given period is

$$
\rho_{S}(\lambda)=\bar{\pi}_{0}\left[1-F_{B}^{*}(V(\lambda))\right] .
$$

This formula takes into account the probability that she is not matched in the period.

A similar expression obtains for $\rho_{B}(\lambda)$, the probability that a buyer submitting bid $\lambda$ successfully trades in any given period. In order to derive this expression, we need a formula for $\omega_{k}(\zeta)$, the probability that the buyer is matched with $k$ rival buyers. If $T_{B}$ is the mass of active buyers and $T_{S}$ is the mass of active sellers, then $\omega_{k}(\zeta) T_{B}$, the mass of buyers participating in matches with $k$ rival buyers, equals $k+1$ times $\pi_{k+1}(\zeta) T_{S}$, the mass of sellers matched with $k+1$ buyers:

$$
\omega_{k}(\zeta) T_{B}=(k+1) \pi_{k+1}(\zeta) T_{S}
$$

Solving, substituting in the formula for $\pi_{k+1}(\zeta)$, and recalling that $\zeta=T_{B} / T_{S}$ shows that $\omega_{k}(\zeta)$ and $\pi_{k}(\zeta)$ are identical:

$$
\omega_{k}(\zeta)=\frac{(k+1)}{\zeta} \pi_{k+1}(\zeta)=\frac{(k+1)}{\zeta} \frac{\zeta^{k+1}}{(k+1) ! e^{\zeta}}=\pi_{k}(\zeta)
$$


The striking implication of this is that the distribution of bids that a buyer must beat is exactly the same distribution of bids that each seller receives when she is matched with at least one buyer.

Turning back to $\rho_{B}$, a buyer who bids $\lambda$ and is the highest bidder has probability $F_{S}(C(\lambda))$ of having his bid accepted. This is just the probability that the seller with whom the buyer is matched will have a low enough reservation price so as to accept his bid. If a total of $j+1$ buyers are matched with the seller with whom the buyer is matched, then he has $j$ competitors and the probability that all $j$ competitors will bid less than $\lambda$ is $\left[F_{B}(V(\lambda))\right]^{j}$. Therefore the probability that the bid $\lambda$ is successful in a particular period is

$$
\begin{aligned}
\rho_{B}(\lambda) & =F_{S}(C(\lambda)) \sum_{j=0}^{\infty} \omega_{j}(\zeta)\left[F_{B}(V(\lambda))\right]^{j} \\
& =F_{S}(C(\lambda)) \sum_{j=0}^{\infty} \pi_{j}(\zeta)\left[F_{B}(V(\lambda))\right]^{j} \\
& =F_{S}(C(\lambda))\left[\pi_{0}+\bar{\pi}_{0} F^{*}(V(\lambda))\right] .
\end{aligned}
$$

\section{Proof of convergence}

Theorem 1 consists of two parts: "the law of one price" part, which given the characterization in Proposition 7, reduces to

$$
\lim _{\delta \rightarrow 0} \underline{c}_{\delta}=\lim _{\delta \rightarrow 0} \bar{p}_{\delta}=\lim _{\delta \rightarrow 0} \underline{v}_{\delta}=p_{W}
$$

and the efficiency part

$$
\lim _{\delta \rightarrow 0} W_{S \delta}(c)=\max \left[0, p_{W}-c\right], \lim _{\delta \rightarrow 0} W_{B \delta}(v)=\max \left[0, v-p_{W}\right]
$$

These are dealt with separately in Propositions 8 and 12 below.

Proposition $8 \lim _{\delta \rightarrow 0} \underline{c}_{\delta}=\lim _{\delta \rightarrow 0} \bar{p}_{\delta}=\lim _{\delta \rightarrow 0} \underline{v}_{\delta}=p_{W}$.

The proof of this Proposition relies on three Lemmas.

Lemma $9 \lim _{\delta \rightarrow 0}\left(\bar{p}_{\delta}-\bar{c}_{\delta}\right)=0$.

Proof. Suppose not, i.e., there exists an $\varepsilon>0$ such that $\bar{p}_{\delta}-\bar{c}_{\delta}>\varepsilon$ along a subsequence. Let

$$
\begin{aligned}
b_{\delta} & =\bar{p}_{\delta}-\varepsilon / 2, \\
v_{\delta} & =\sup \left\{v: B_{\delta}(v) \leq b_{\delta}\right\} .
\end{aligned}
$$

Let the probability $\gamma_{\delta}$ be the seller's equilibrium belief that the maximum bid in a given period is greater than or equal to $b_{\delta}$. If $\lim _{\delta \rightarrow 0} \gamma_{\delta}=\gamma>0$ along a subsequence, the seller for whom $c_{\delta}=\left(\bar{c}_{\delta}+b_{\delta}\right) / 2$ would prefer to enter for small enough $\delta$. The reason is this. By definition $b_{\delta}-\bar{c}_{\delta}>\varepsilon / 2$ and, therefore, $b_{\delta}-c_{\delta}>\varepsilon / 4$. Consequently, if seller 


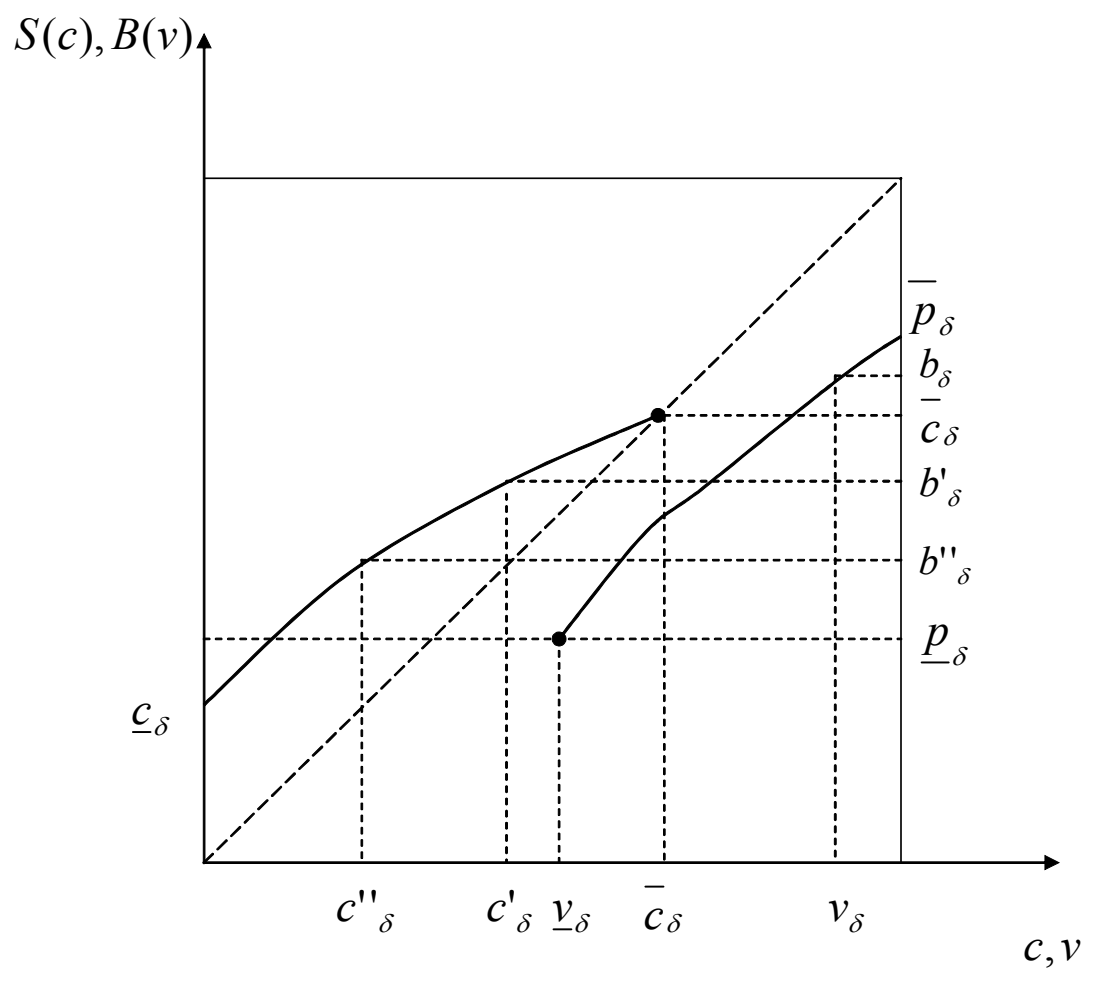

Figure 2: Construction of $b^{\prime}, b^{\prime \prime}, c_{\delta}^{\prime}$ and $c_{\delta}^{\prime \prime}$ used in the proof of Theorem 1 
$c_{\delta}$ sets her offer to be $\lambda=b_{\delta}$, then the gain $U$ she realizes if she trades is at least $\varepsilon / 4$ and her per period probability of trade is $\rho_{S}(\lambda)>\underline{\gamma}$. Inspection of formulas (7) and (11) establishes that as $\delta \rightarrow 0$ her discounted probability of trade goes to 1 and her discounted participation costs goes to 0 . Therefore her expected utility, as given by $(10)$, is

$$
W^{S}(\lambda, U)=P_{S}(\lambda) U-K_{S}(\lambda)>\frac{\varepsilon}{4}>0
$$

as $\delta \rightarrow 0$, a contradiction.

If, on the other hand, $\lim _{\delta \rightarrow 0} \gamma_{\delta}=0$ along all subsequences, then the buyer for whom $v=1$ would prefer a deviation to $b_{\delta}$. If he deviates, then in the limit, as $\delta \rightarrow 0$, his probability of trading in a given period, $\rho_{B}\left(b_{\delta}\right)$, approaches 1 . This is an immediate implication of the observation that follows (18): $\gamma_{\delta}$ is not only the probability that the maximum bid a seller receives in a given period is greater than or equal to $b_{\delta}$, but it is also the probability that the maximum competing bid the type 1 buyer must beat is greater than or equal to $b_{\delta}$. Therefore $\gamma_{\delta} \rightarrow 0$ implies that deviating to $b_{\delta}$ results in his discounted probability approaching 1 and discounted participation cost approaching 0 . Consequently, this buyer deviates and secures the lower price $b_{\delta}$, which completes the Lemma's proof.

Lemma $10 \lim _{\delta \rightarrow 0}\left(\bar{p}_{\delta}-\underline{p}_{\delta}\right)=0$.

The proof is by contradiction: pick a small $\varepsilon$, suppose $\bar{p}_{\delta}-\underline{p}_{\delta}>\varepsilon>0$ along a subsequence, and define

$$
\begin{aligned}
& b_{\delta}^{\prime}=\bar{p}_{\delta}-\frac{1}{3} \varepsilon, \\
& b_{\delta}^{\prime \prime}=\bar{p}_{\delta}-\frac{2}{3} \varepsilon .
\end{aligned}
$$

Note that $b_{\delta}^{\prime \prime}-\underline{p}_{\delta}>\frac{\varepsilon}{3}$. Select a buyer and let

$$
\phi_{\delta}=F_{S \delta}\left(C_{\delta}\left(b_{\delta}^{\prime}\right)\right)
$$

be the equilibrium probability that the seller with whom he is matched in a given period would accept a bid that is less than or equal to $b_{\delta}^{\prime}$. Lemma 9 guarantees that the seller for whom $S(c)=b_{\delta}^{\prime}$ exists, at least for small enough $\delta$. Select a seller and let

$$
\begin{aligned}
\psi_{\delta} & =\sum_{k=0}^{\infty} \pi_{k}\left[F_{B \delta}\left(V_{\delta}\left(b_{\delta}^{\prime}\right)\right)\right]^{k} \\
& =\pi_{0}+\bar{\pi}_{0} F_{B \delta}^{*}\left(V_{\delta}\left(b_{\delta}^{\prime}\right)\right) .
\end{aligned}
$$

be the equilibrium probability that, in a given period, she receives either no bid or the highest bid she receives is less or equal to $b_{\delta}^{\prime}$. Observe that $\psi_{\delta}$ is the equilibrium probability that a buyer's bid $b_{\delta}^{\prime}$ is maximal in a given match; this follows directly from formula (19) for $\rho_{B}(\lambda)$. Given these definitions, this Lemma's proof consists of three steps. 
Step 1. The fraction of sellers for whom $S_{\delta}(c) \leq b_{\delta}^{\prime}$ does not vanish as $\delta \rightarrow 0$, i.e., $\underline{\phi} \equiv \underline{\lim }_{\delta \rightarrow 0} \phi_{\delta}>0$.

Suppose not. Then $\phi_{\delta} \rightarrow 0$ along a subsequence. Fix this subsequence and fix some period, say period 0 . Let $N_{\delta}$ be a sequence of integers whose values are chosen later in the proof. Define, without loss of generality, the time segment $\Upsilon_{\delta}$ of length $N_{\delta}$ periods that begins with period 0 and ends with period $N_{\delta}$. Define three masses of sellers :

- $m_{N \delta}^{+}$is the mass of sellers who enter the market within time segment $\Upsilon_{\delta}$ and for whom $b_{\delta}^{\prime \prime} \leq S_{\delta}(c) \leq b^{\prime}$.

- $m_{N \delta}^{-}$is the mass of sellers who both enter and exit the market within time segment $\Upsilon_{\delta}$ and for whom $b_{\delta}^{\prime \prime} \leq S_{\delta}(c) \leq b_{\delta}^{\prime}$.

- $m_{\delta}$ the steady-state mass of active sellers for whom $b_{\delta}^{\prime \prime} \leq S_{\delta}(c) \leq b_{\delta}^{\prime}$.

The assumption that $\phi_{\delta} \rightarrow 0$ implies that $m_{\delta} \rightarrow 0$. We show next that $m_{\delta} \rightarrow 0$ entails $\underline{c}_{\delta} \rightarrow b_{\delta}^{\prime \prime}$. This establishes a contradiction because Proposition 7 states that $\underline{c}_{\delta}<\underline{p}_{\delta}$ and by construction $\underline{p}_{\delta}+\frac{\varepsilon}{3}<b_{\delta}^{\prime \prime}$.

The fraction of sellers in the mass $m_{N \delta}^{+}$that do not exit during the time segment $\Upsilon_{\delta}$ is

$$
\frac{m_{N \delta}^{+}-m_{N \delta}^{-}}{m_{N \delta}^{+}} \leq \frac{m_{\delta}}{m_{N \delta}^{+}}
$$

because the surviving mass $m_{N \delta}^{+}-m_{N \delta}^{-}$of sellers who entered in time segment $\Upsilon_{\delta}$ cannot exceed the total, steady state of the mass $m_{\delta}$ of sellers with reservation prices in the interval $\left[b_{\delta}^{\prime \prime}, b_{\delta}^{\prime}\right]$. Therefore the fraction of the sellers in $m_{N \delta}^{+}$that have traded within time segment $\Upsilon_{\delta}$ is at least

$$
1-\frac{m_{\delta}}{m_{N \delta}^{+}}
$$

In the mass $m_{N \delta}^{+}$, pick a seller $c_{\delta}^{\prime \prime}$ who enters in period 0 and for whom

$$
S_{\delta}\left(c_{\delta}^{\prime \prime}\right)=b_{\delta}^{\prime \prime}
$$

Such a seller $c_{\delta}^{\prime \prime}$ always exists because $S_{\delta}$ is continuous (see Proposition 7 ) and $\underline{g}$ is a lower bound on the density of entering sellers. This seller has as low reservation price as any other seller in $m_{N \delta}^{+}$and has the full time segment $\Upsilon_{\delta}$ in which to consummate a trade. She therefore has as high probability of trading within $\Upsilon_{\delta}$ as any other seller in $m_{N \delta}^{+}$. Let $r_{\delta}$ be her probability of trading within the time segment $\Upsilon_{\delta}$. It is therefore at least as great as the average probability of trading across all sellers in $m_{N \delta}^{+}$:

$$
r_{\delta} \geq 1-\frac{m_{\delta}}{m_{N \delta}^{+}}
$$

Now, since the slope of $S_{\delta}$ is at most one (see the formula in Proposition 7), it follows that

$$
m_{N \delta}^{+} \geq \frac{\varepsilon}{3} \underline{g} \delta N_{\delta}
$$


because $m_{N \delta}^{+}$is minimized when the slope of $S_{\delta}$ is the largest (i.e., equal to 1 ) and the density $g_{S}$ is minimal. Substituting this lower bound on $m_{N \delta}^{+}$into (22) gives

$$
r_{\delta} \geq 1-\frac{m_{\delta}}{\frac{\varepsilon}{3} \underline{g} \delta N_{\delta}} .
$$

For seller $c_{\delta}^{\prime \prime}$ her discounted probability of trading $P_{S \delta}\left(b_{\delta}^{\prime \prime}\right)$ from setting reservation price $b_{\delta}^{\prime \prime}$ is bounded from below by

$$
P_{S \delta}\left(b_{\delta}^{\prime \prime}\right) \geq e^{-\beta \delta N_{\delta}}\left(1-\frac{m_{\delta}}{\frac{\varepsilon}{3} \underline{g} \delta N_{\delta}}\right) .
$$

The right-hand side understates the discounted probability of trade because, literally, the lower bound is the discounted probability of trader $c_{\delta}^{\prime \prime}$ waiting the full $N_{\delta}$ periods before attempting to trade, having only probability $1-m_{\delta} /\left(\frac{\varepsilon}{3} \underline{g} \delta N_{\delta}\right)$ of succeeding in that period, and then never trying again.

Set the period length to be

$$
N_{\delta}=\min \left\{k: k \text { is integer, } k \geq \frac{\sqrt{m_{\delta}}}{\delta}\right\} .
$$

Substitution of this choice into (24) and taking the limit as $\delta \rightarrow 0$ shows that discounted probability of seller $c_{\delta}^{\prime \prime}$ trading approaches 1 from below because $m_{\delta} \rightarrow 0$ :

$$
\lim _{\delta \rightarrow 0} P_{S \delta}\left(S_{\delta}\left(c_{\delta}^{\prime}\right)\right) \geq \lim _{\delta \rightarrow 0} \exp \left(-\beta \sqrt{m_{\delta}}\right)\left(1-\frac{\sqrt{m_{\delta}}}{\frac{\varepsilon}{3} \underline{g}}\right)=1 .
$$

Recall from Proposition 7 that, for almost all $c \in[0, \bar{c})$,

$$
S_{\delta}^{\prime}(c)=1-e^{-\beta \delta} P_{S \delta}\left[S_{\delta}(c)\right] .
$$

Since $P_{S \delta}\left(S_{\delta}(c)\right) \geq P_{S \delta}\left(b_{\delta}^{\prime \prime}\right)$ for $c \leq b_{\delta}^{\prime \prime}$ and $S_{\delta}$ is increasing on $[0, \bar{c})$, it follows that, for all seller types $c \in\left[0, c_{\delta}^{\prime \prime}\right], P_{S \delta}\left[S_{\delta}(c)\right] \rightarrow 1$ and

$$
\lim _{\delta \rightarrow 0} S_{\delta}^{\prime}(c)=0
$$

Consequently, since $S_{\delta}$ is continuous,

$$
\underline{c}_{\delta}=S_{\delta}(0) \rightarrow b_{\delta}^{\prime \prime}
$$

This is in contradiction to $\underline{c}_{\delta}<\underline{p}_{\delta}<b_{\delta}^{\prime \prime}-\varepsilon / 3$. Therefore it can not be that $\phi_{\delta} \rightarrow 0$.

Step 2. If the ratio of buyers to sellers $\zeta_{\delta}$ is bounded away from 0 , then the probability $\psi_{\delta}$ that the highest bid in a given meeting is less than $b_{\delta}^{\prime}$ is also bounded away from 0 . Proof of this step stands alone and is not based on the result in step 1 of this proof.

Formally, if $\underline{\lim }_{\delta \rightarrow 0} \zeta_{\delta}>0$, then $\underline{\psi} \equiv \underline{\lim }_{\delta \rightarrow 0} \psi_{\delta}>0$. Suppose not. Then $\psi_{\delta} \rightarrow 0$ and $\zeta_{\delta} \rightarrow \underline{\zeta}>0$ along a subsequence. Fix this subsequence and recall that by construction

$$
b_{\delta}^{\prime \prime}>\underline{p}_{\delta}+\frac{\varepsilon}{3} .
$$


First, we show that the seller with cost $c_{\delta}^{\prime \prime}$ such that $S\left(c_{\delta}^{\prime \prime}\right)=b_{\delta}^{\prime \prime}$ prefers to enter. Since $\zeta_{\delta} \rightarrow \underline{\zeta}$ and $\psi_{\delta} \rightarrow 0$, for all $\delta$ sufficiently small, the probability that he meets a buyer for whom $B(v) \geq b_{\delta}^{\prime}=b_{\delta}^{\prime \prime}+\varepsilon / 3$ is at least $\frac{1}{2}\left(1-e^{-\underline{\zeta}}\right)$. This is because, with $\psi_{\delta} \rightarrow 0$, (i) almost every bid she receives is greater than $b_{\delta}^{\prime}$ and (ii) her probability of getting at least one bid is approaching $1-e^{-\underline{\zeta}}$. Therefore, as $\delta \rightarrow 0$ her discounted probability of trading with a buyer for whom $B_{\delta}(v) \geq b_{\delta}^{\prime}$ approaches 1 even as her discounted participation costs, given by formula (9), approach 0 . Consequently, the profit of the $c_{\delta}^{\prime \prime}$ seller, in the limit as $\delta \rightarrow 0$, is at least $\varepsilon / 3$, and she will choose to enter.

Second, since she chooses to enter, it must be that $c_{\delta}^{\prime \prime} \leq \bar{c}_{\delta}$. Therefore the slope of $S$ for $c \in\left[0, c_{\delta}^{\prime \prime}\right)$ satisfies

$$
S^{\prime}(c)=1-e^{-\beta \delta} P_{S \delta}(c) \rightarrow 0
$$

since $P_{S \delta}\left(S_{\delta}(c)\right) \geq P_{S \delta}\left(S_{\delta}\left(c_{\delta}^{\prime \prime}\right)\right)$ and $P_{S \delta}\left(S_{\delta}\left(c_{\delta}^{\prime \prime}\right)\right) \rightarrow 1$. Therefore $\underline{c}_{\delta} \rightarrow b_{\delta}^{\prime \prime}$, a contradiction of (25) and Proposition 7's requirement that $\underline{c}_{\delta}<\underline{p}_{\delta}$.

Step 3. For small enough $\delta$, a buyer for whom $v=1$ prefers to deviate to bidding $b_{\delta}^{\prime}$ instead of $\bar{p}_{\delta}$. There are two cases to consider.

Case 1. $\underline{\lim }_{\delta \rightarrow 0} \zeta_{\delta}>0$. We show, using both steps 1 and 2 of this proof, that bidding $\bar{p}_{\delta}$ cannot be equilibrium behavior for a type 1 buyer. Recall that $\phi_{\delta}$ is the probability that a seller will accept a bid less than $b_{\delta}^{\prime}$ and that, according to step 1, $\phi=\lim _{\delta \rightarrow 0} \phi_{\delta}>0$. Additionally, recall that $\psi_{\delta}$ is the probability that the maximal rival bid a buyer faces in a given period is no greater than $b_{\delta}^{\prime}$ and that, according to step $2, \lim _{\delta \rightarrow 0} \psi_{\delta}=\psi>0$. For small enough $\delta>0$, this second probability is bounded from below by $(1 / 2) \bar{\psi}$. It follows that, for small enough $\delta$, the buyer who bids $b_{\delta}^{\prime}$ (i) wins over all his rival buyers with probability greater than $(1 / 2) \psi$, and (ii) has his bid accepted by the seller with probability greater than $(1 / 2) \underline{\phi}$. Therefore, as $\delta \rightarrow 0$, the buyer who bids $b_{\delta}^{\prime}$ trades with a discounted probability approaching 1 and a discounted participation cost approaching 0 . Consequently deviating to $b_{\delta}^{\prime}$ gives him a profit of at least $1-b_{\delta}^{\prime}$, which is greater than $1-\bar{p}_{\delta}$, that profit he would make with his equilibrium bid $B(1)=\bar{p}_{\delta}$. Therefore deviation to $b_{\delta}^{\prime}$ is profitable for him.

Case 2. $\varliminf_{\delta \rightarrow 0} \zeta_{\delta}=0$. Fix a subsequence such that $\zeta_{\delta} \rightarrow 0$. The proof of this case relies only on the result in step 1 of this proof. The probability of meeting no rival buyers in a given period is $e^{-\zeta_{\delta}}$ and, since $\zeta_{\delta} \rightarrow 0$, this probability is at least $1 / 2$ for sufficiently small $\delta$. In any given period, for a type 1 buyer and for all small $\delta$ and (i) the probability of meeting no rivals is at least $1 / 2$ and (ii) the probability of meeting a seller who would accept the bid $b_{\delta}^{\prime}$ is at least $(1 / 2) \phi$. It follows that as $\delta \rightarrow 0$, his discounted probability of trading approaches 1 and his discounted participation cost approaches 0 . Therefore deviating to $b_{\delta}^{\prime}$ gives him a profit of at least $1-b_{\delta}^{\prime}>1-\bar{p}_{\delta}$, which proves that a deviation to $b_{\delta}^{\prime}$ is profitable for him.

Step 3 completes the Lemma's proof because it contradicts the hypothesis that, $\lim _{\delta \rightarrow 0}\left(\bar{p}_{\delta}-\underline{p}_{\delta}\right)=\varepsilon>0$

Lemma $11 \lim _{\delta \rightarrow 0}\left(\underline{v}_{\delta}-\underline{p}_{\delta}\right)=0$. 
Proof. Suppose not. Recall that $B\left(\underline{v}_{\delta}\right) \equiv \underline{p}_{\delta}$ and that Proposition 7 states that $\underline{v}_{\delta}>\underline{p}_{\delta}$. Pick a subsequence such that $\underline{v}_{\delta}-\underline{p}_{\delta} \geq \eta>0$ along it. Define $\xi_{\delta}=\frac{1}{2}\left(\underline{p}_{\delta}+\underline{v}_{\delta}\right)$ and observe that $\xi_{\delta}-\underline{p}_{\delta} \geq \eta / 2$ and $\xi_{\delta}<\underline{v}_{\delta}$. The latter inequality implies that a type $\xi_{\delta}$ buyer does not enter the market because his expected utility is non-positive. But suppose to the contrary that a type $\xi_{\delta}$ buyer enters and bids $\bar{p}_{\delta}$. Bidding $\bar{p}_{\delta}$ guarantees that he wins the auction in whatever match he finds himself, i.e., $\rho_{B \delta}\left(\bar{p}_{\delta}\right)=1$. Therefore in the first period after he enters he earns profit of

$$
\begin{aligned}
& \xi_{\delta}-\bar{p}_{\delta}-\kappa \delta \\
= & \xi_{\delta}-\underline{p}_{\delta}+\underline{p}_{\delta}-\bar{p}_{\delta}-\kappa \delta \\
\geq & \frac{\eta}{2}+\underline{p}_{\delta}-\bar{p}_{\delta}-\kappa \delta \\
\rightarrow & \frac{\eta}{2}
\end{aligned}
$$

because Lemma 10 states that, as $\delta \rightarrow 0, \bar{p}_{\delta}-\underline{p}_{\delta} \rightarrow 0$. This contradicts the equilibrium decision of the type $\xi_{\delta}$ buyer not to enter.

Proof of Proposition 8. Consider any sequence of equilibria $\delta_{n} \rightarrow 0$. The descriptors $\bar{p}_{\delta}$ and $\underline{v}_{\delta}$ converge because

$$
\begin{aligned}
\lim _{\delta \rightarrow 0}\left(\bar{p}_{\delta}-\underline{v}_{\delta}\right) & =\lim _{\delta \rightarrow 0}\left(\bar{p}_{\delta}-\underline{v}_{\delta}\right)-\lim _{\delta \rightarrow 0}\left(\underline{p}_{\delta}-\underline{v}_{\delta}\right) \\
& =\lim _{\delta \rightarrow 0}\left(\bar{p}_{\delta}-\underline{p}_{\delta}\right) \\
& =0
\end{aligned}
$$

where $\lim _{\delta \rightarrow 0}\left(\underline{p}_{\delta}-\underline{v}_{\delta}\right)=0$ (from Lemma 11) implies the first equality and $\lim _{\delta \rightarrow 0}\left(\bar{p}_{\delta}-\right.$ $\left.\underline{p}_{\delta}\right)=0$ (from Lemma 10) implies the third equality. Proposition 7 establishes that $\overline{\bar{c}}_{\delta} \in\left[\underline{p}_{\delta}, \bar{p}_{\delta}\right)$; therefore Lemma 10 implies

$$
\lim _{\delta \rightarrow 0}\left(\bar{p}_{\delta}-\bar{c}_{\delta}\right)=0
$$

Pick a convergent subsequence of $\left(\underline{v}_{\delta}, \bar{p}_{\delta}, \bar{c}_{\delta}, \underline{p} \delta\right)$ and denote its limit as $\left(p_{*}, p_{*}, p_{*}, p_{*}\right)$.

Traders who choose to become active in the market exit only by trading. Therefore in the steady state the mass of sellers entering each period must equal the mass of buyers entering each period:

$$
G_{S}\left(\bar{c}_{\delta}\right)=a \bar{G}_{B}\left(\underline{v}_{\delta}\right) .
$$

Taking the limit in (28) along the convergent subsequence as $\delta \rightarrow 0$, we get

$$
G_{S}\left(p_{*}\right)=a \bar{G}_{B}\left(p_{*}\right) .
$$

This is just equation (1) that defines the Walrasian price; therefore $p_{*}=p_{W}$. Since $p_{W}$ is the common limit of all convergent subsequences, it follows that the original sequence $\left(\underline{v}_{\delta}, \bar{p}_{\delta}, \bar{c}_{\delta}, \underline{p}_{\delta}\right)$ converges to the same limit:

$$
\lim _{\delta \rightarrow 0} \bar{p}_{\delta}=\lim _{\delta \rightarrow 0} \underline{p}_{\delta}=\lim _{\delta \rightarrow 0} \bar{c}_{\delta}=\lim _{\delta \rightarrow 0} \underline{v}_{\delta}=p_{W}
$$


All that remains is to show that $\underline{c}_{\delta}$ also converges to $p_{W}$. The type $\bar{c}_{\delta}$ seller who is on the margin between participating and not participating must in expectation be just recovering his participation cost each period. Recall that $S_{\delta}\left(\bar{c}_{\delta}\right)=\bar{c}_{\delta}$. Since the price this seller receives is no more than the highest bid, $\bar{p}_{\delta}$, it follows that

$$
\rho_{S \delta}\left[S_{\delta}\left(\bar{c}_{\delta}\right)\right]\left(\bar{p}_{\delta}-\bar{c}_{\delta}\right) \geq \kappa \delta .
$$

Therefore

$$
\frac{\rho_{S \delta}\left[S_{\delta}\left(\bar{c}_{\delta}\right)\right]}{\delta} \geq \frac{\kappa}{\bar{p}_{\delta}-\bar{c}_{\delta}} \rightarrow \infty
$$

by (27). The discounted probability of trade may be written as

$$
\begin{aligned}
P_{S \delta}\left[S_{\delta}\left(\bar{c}_{\delta}\right)\right] & =\frac{\rho_{S \delta}\left[S_{\delta}\left(\bar{c}_{\delta}\right)\right]}{1-e^{-\beta \delta}+e^{-\beta \delta} \rho_{S}\left[S_{\delta}\left(\bar{c}_{\delta}\right)\right]} \\
& =\frac{1}{\frac{\frac{1-e^{-\beta \delta}}{\delta}}{\frac{\left.\rho_{S \delta} S_{\delta}\left(\bar{c}_{\delta}\right)\right]}{\delta}}+e^{-\beta \delta}} .
\end{aligned}
$$

It follows that $\lim _{\delta \rightarrow 0} P_{S}\left[S_{\delta}\left(\bar{c}_{\delta}\right)\right]=1$ because $\lim _{\delta \rightarrow 0} \frac{1-e^{-\beta \delta}}{\delta}=\beta$ and $\lim _{\delta \rightarrow 0} \frac{\rho_{S \delta}\left[S_{\delta}\left(\bar{c}_{\delta}\right)\right]}{\delta}=$ $\infty$. Further, for all $c \in\left[0, \bar{c}_{\delta}\right]$,

$$
\lim _{\delta \rightarrow 0} P_{S \delta}\left[S_{\delta}\left(c_{\delta}\right)\right]=1
$$

because $P_{S \delta}\left[S_{\delta}(\cdot)\right]$ is decreasing. Therefore

$$
S_{\delta}^{\prime}(c)=1-e^{-\beta \delta} P_{S \delta}\left(S_{\delta}(c)\right),
$$

the slope of $S_{\delta}$ on $\left[0, \bar{c}_{\delta}\right]$, converges to 0 . Together with the continuity of $S_{\delta}$ this implies that $\underline{c}_{\delta} \rightarrow \bar{c}_{\delta}$, which completes the proof of the proposition.

The next Proposition proves the second part of Theorem 1.

Proposition $12 \lim _{\delta \rightarrow 0} W_{S \delta}(c)=\max \left[0, p_{W}-c\right]$ and $\lim _{\delta \rightarrow 0} W_{B \delta}(v)=\max \left[0, v-p_{W}\right]$.

Proof. Equation (31) establishes that, for all $c \in\left[0, \bar{c}_{\delta}\right], \lim _{\delta \rightarrow 0} P_{S \delta}\left[S_{\delta}\left(\bar{c}_{\delta}\right)\right]=1$. The same argument, slightly adapted, shows that, for all $v \in\left[\underline{v}_{\delta}, 1\right], \lim _{\delta \rightarrow 0} P_{B \delta}\left[B_{\delta}(v)\right]=$ 1. Thus the buyer for whom $v=\underline{v}_{\delta}$ must just recover its participation cost each period:

$$
\rho_{B \delta}\left[B_{\delta}\left(\underline{v}_{\delta}\right)\right]\left(\underline{v}_{\delta}-B_{\delta}\left(\underline{v}_{\delta}\right)\right)=\rho_{B \delta}\left(\underline{p}_{\delta}\right)\left(\underline{v}_{\delta}-\underline{p}_{\delta}\right)=\kappa \delta .
$$

Therefore

$$
\frac{\rho_{B \delta}\left(\underline{p}_{\delta}\right)}{\delta}=\frac{\kappa}{\underline{v}_{\delta}-\underline{p}_{\delta}} \rightarrow \infty
$$

by Lemma 11 and, exactly as with (30),

$$
\lim _{\delta \rightarrow 0} P_{B \delta}\left(\underline{p}_{\delta}\right)=\lim _{\delta \rightarrow 0} \frac{\rho_{B \delta}\left(\underline{p}_{\delta}\right)}{1-e^{-\beta \delta}+e^{-\beta \delta} \rho_{B \delta}\left(\underline{p}_{\delta}\right)}=1
$$


Since $P_{B \delta}(\cdot)$ is increasing, this establishes that $\lim _{\delta \rightarrow 0} P_{B \delta}\left[B_{\delta}\left(v_{\delta}\right)\right]=1$ for all $v_{\delta} \in$ $\left[\underline{v}_{\delta}, 1\right]$.

The envelope theorem - see equations (12) and (14) —implies that

$$
\begin{aligned}
W_{S \delta}(c) & =\int_{c}^{\bar{c}_{\delta}} P_{S \delta}\left[S_{\delta}(x)\right] d x, \\
W_{B \delta}(v) & =\int_{\underline{v}_{\delta}}^{v} P_{B \delta}\left[B_{\delta}(x)\right] d x .
\end{aligned}
$$

Passing to the limit as $\delta \rightarrow 0$ gives $\lim _{\delta \rightarrow 0} W_{S \delta}(c)=\max \left[0, p_{W}-c\right]$ and $\lim _{\delta \rightarrow 0} W_{B \delta}(v)=$ $\max \left[0, v-p_{W}\right]$ because $\bar{c}_{\delta} \rightarrow p_{W}$ and $\underline{v}_{\delta} \rightarrow p_{W}$

\section{$5 \quad$ Full trade equilibria and existence}

Recall that Proposition 7 shows that every equilibrium must satisfy $B(\underline{v}) \leq S(\bar{c})$. The intuition for this is that the type $\underline{v}$ buyer can only trade if there is no rival buyer. Without competition he should certainly not bid more than $S(\bar{c})$, the minimum bid that is always accepted by any seller. If, in fact, $B(\underline{v})=S(\bar{c})$, then the type $\bar{c}$ seller, who among active sellers is the seller with the highest cost, always trades if she is matched with at least one buyer, even if he is the lowest valued active buyer. This, of course, means both that any seller with cost less than $\bar{c}$ also trades if she is matched and that a buyer fails to trade only because he is beaten in the bidding by another buyer. We call an equilibrium of this kind a full trade equilibria because every match results in a trade. In this section we characterize full-trade equilibria and prove their existence for all sufficiently small $\delta>0$. Proposition 13 proves that the full-trade equilibria are fully specified by the vector of parameters $(\bar{c}, \underline{v}, \zeta)$. Figure 3 illustrates an equilibrium of this special type. Proposition 14 proves that a unique full-trade equilibrium exists for all sufficiently small $\delta>0$. Theorem 2 is then a corollary of Proposition 14.

Before introducing the equations that determine $(\bar{c}, \underline{v}, \zeta)$, we derive sellers and buyers' probabilities of trade as a function of their types $c$ and $v$ and the buyer-seller ratio $\zeta$. As a consequence of the equilibrium being full trade buyers' trade probabilities are independent of sellers' equilibrium strategy $S$. That the sellers' strategy does not feed back and affect the buyers' trade probabilities and strategy implies that the market fundamentals - $G_{S}, G_{B}, a, \kappa, \beta$, and $\delta$-fully determine the equilibrium. This fact drives both the uniqueness and existence results of this section.

Given that the market is in a steady state, within every period the cohort of buyers who has the highest valuations in their matches and therefore trades is replaced by an entering cohort of equal size and composition. Therefore $F_{B}^{*}$, the distribution function of the maximal valuation within a match, is equal to the distribution of $v$ in the entering cohort conditional on $v \geq \underline{v}$ :

$$
F_{B}^{*}(v)=\frac{G_{B}(v)-G_{B}(\underline{v})}{1-G_{B}(\underline{v})} .
$$

Let $\hat{\rho}_{B}(v)$ be the probability that a type $v$ buyer trades in any given period. It, as reference back to equation (19) and its derivation explains, is equal to the probability 
that he bids against no rival buyers $\left(\omega_{0}=e^{-\zeta}\right)$ plus the complementary probability $\left(\bar{\omega}_{0}=1-e^{-\zeta}\right)$ times the probability that the maximal value among the rival buyers in his match is no greater than $v:^{9}$

$$
\hat{\rho}_{B}(v)=e^{-\zeta}+\left(1-e^{-\zeta}\right) F_{B}^{*}(v) .
$$

The discounted trading probability of a type $v$ buyer is therefore

$$
\hat{P}_{B}(v)=\frac{\hat{\rho}_{B}(v)}{1-e^{-\beta \delta}+e^{-\beta \delta} \hat{\rho}_{B}(v)} .
$$

The hats on $\hat{\rho}_{B}(\cdot)$ and $\hat{P}_{B}(\cdot)$ emphasize that these probabilities are functions of the buyer's value $v$, not of his bid $B(v)$.

With this notation in place we can introduce the equations that determine $(\bar{c}, \underline{v}, \zeta)$ in a full trade equilibrium. First, since every meeting results in a trade, the mass of entering buyers must equal the mass of entering sellers in the steady state:

$$
\begin{aligned}
G_{S}(\bar{c}) & =a\left[1-G_{B}(\underline{v})\right] \\
& =a \bar{G}(\underline{v}) .
\end{aligned}
$$

Second, the buyer for whom $v=\underline{v}$ must be indifferent between being active and staying out of the market. The type $\underline{v}$ buyer only trades in a period when there are no rival buyers; his probability of trading is $\omega_{0}=e^{-\zeta}$. Since in a full-trade equilibrium $B(\underline{v})=$ $S(\bar{c})$ and Proposition 7 states that $S(\bar{c})=\bar{c}$, indifference necessarily implies that his expected gains from trade in any period, $(\underline{v}-\bar{c}) e^{-\zeta}$ equals his participation cost:

$$
(\underline{v}-\bar{c}) e^{-\zeta}=\kappa \delta \text {. }
$$

Third, parallel logic applies to any seller for whom $c=\bar{c}$. This seller always trades in any period in which he is matched with at least one buyer; the probability of this event is $1-\pi_{0}=\bar{\pi}_{0}=1-e^{-\zeta}$. Denote the expected price that any seller receives as $p$. Note that this expected price is not a function of the sellers' type $c$; it is the same for all active sellers in a full-trade equilibrium. Then, since the $\bar{c}$ seller is indifferent between trading and staying out of the market, it must be that

$$
(p-\bar{c})\left(1-e^{-\zeta}\right)=\kappa \delta .
$$

In order to find the price $p$, we use the envelope theorem to solve for the bidding strategies of the active buyers (i.e., those buyers for whom $v \geq \underline{v}$ ) as follows:

$$
\begin{aligned}
W_{B}(v) & =(v-B(v)) \hat{P}_{B}(v)-K_{0}(v) \\
& =\int_{\underline{v}}^{v} \hat{P}_{B}(x) d x
\end{aligned}
$$

\footnotetext{
${ }^{9}$ Formula (19),

$$
\rho_{B}(\lambda)=F_{S}(C(\lambda))\left[\pi_{0}+\bar{\pi}_{0} F^{*}(V(\lambda))\right],
$$
}

illustrates why the full trade case is different than the general case. In the full trade case the factor $F_{S}(C(\lambda))$ is degenerate: for all $\lambda \in[\underline{p}, \bar{p}], F_{S}(C(\lambda))=1$. As a consequence the seller's inverse strategy $C(\lambda)$ does not affect $\rho_{B}(\cdot)$. In the general case, an interval $[\underline{p}, \underline{\lambda}] \subset[\underline{p}, \bar{p}]$ may exist such that, for all $\lambda \in[\underline{p}, \underline{\lambda}], F_{S}(C(\lambda))<1$ and $C(\lambda)$ does affect $\rho_{B}(\cdot)$. 
where $\hat{P}_{B}(v)$ is the type $v$ buyer's discounted probability of trading and

$$
K_{0}(v)=\frac{\kappa \delta}{1-e^{-\beta \delta}+e^{-\beta \delta} \hat{\rho}_{B}(v)}
$$

is his discounted participation cost. Solving equation (38) for $B(v)$ gives

$$
B(v)=v-\frac{\kappa \delta}{\hat{\rho}_{B}(v)}-\frac{1}{\hat{P}_{B}(v)} \int_{\underline{v}}^{v} \hat{P}_{B}(x) d x .
$$

Observe that this formula calculates $B(v)$ directly; it is not a fixed point condition. The expected price $p$ that a seller receives is the expected value of $B(v)$ for that buyer who has the highest valuation:

$$
p=\int_{\underline{v}}^{1} B(v) d F_{B}^{*}(v)=\frac{1}{1-G_{B}(\underline{v})} \int_{\underline{v}}^{1} B(v) d G_{B}(v)
$$

where the second equality follows from equation (32).

Equations (35-37) form a system of three equations in three unknowns $(\bar{c}, \underline{v}, \zeta)$ that, for given $\delta$, must hold in any full-trade equilibrium. In Proposition 13 below we prove that the converse claim is also true: there is a unique full-trade equilibrium that corresponds to any given solution $(\bar{c}, \underline{v}, \zeta)$. The system $(35-37)$ - the characterizing equations - therefore identifies a full-trade equilibrium.

Proposition 13 For given $\delta>0$, any full-trade equilibrium must satisfy conditions $(35-37)$. Conversely, for given $\delta>0$, any solution $(\bar{c}, \underline{v}, \zeta) \in(0,1)^{2} \times(0, \infty)$ to $(35$ 37) corresponds to a unique full-trade equilibrium.

Proof. We have already seen that equations (35-37) must hold in any full-trade equilibrium. We also know that the strategies $S$ and $B$ are strictly increasing. It remains to prove the second part of the Proposition that, for given $\delta>0$ and solution $(\bar{c}, \underline{v}, \zeta) \in(0,1)^{2} \times(0, \infty)$ to system (35-37), a unique full-trade equilibrium exists. To show this we need to start with the solution values $(\bar{c}, \underline{v}, \zeta)$, construct the unique steady-state densities $f_{B}$ and $f_{S}$ and strategies $B$ and $S$, compute the masses $T_{B}$ and $T_{S}$ that these densities and strategies imply, and check that the ratio of these masses equals the solution value $\zeta$. The key insight to our construction is the observation that the strategy for buyers can be constructed separately from the strategy for sellers; the solution $(\bar{c}, \underline{v}, \zeta)$ is a sufficient link between the two.

Consider buyers first. The lowest active buyer's type, $\underline{v}$, is a component of the characterizing equations' solution. The steady-state distribution, $F_{B}^{*}$, of the maximal rival buyer's type is then given by formula (32). The constraint simplification theorem in Milgrom (2004, p. 105) implies that the envelope condition (38) is sufficient for $B$ to be a best-response. Buyers' unique symmetric mutual best-response strategy for $v \geq \underline{v}$ is therefore given by (39). For $v<\underline{v}$, the best-response is not to enter. Observe that the formula implies, as it should, that $B$ is an increasing function and that $B(\underline{v})=\bar{c}$. 


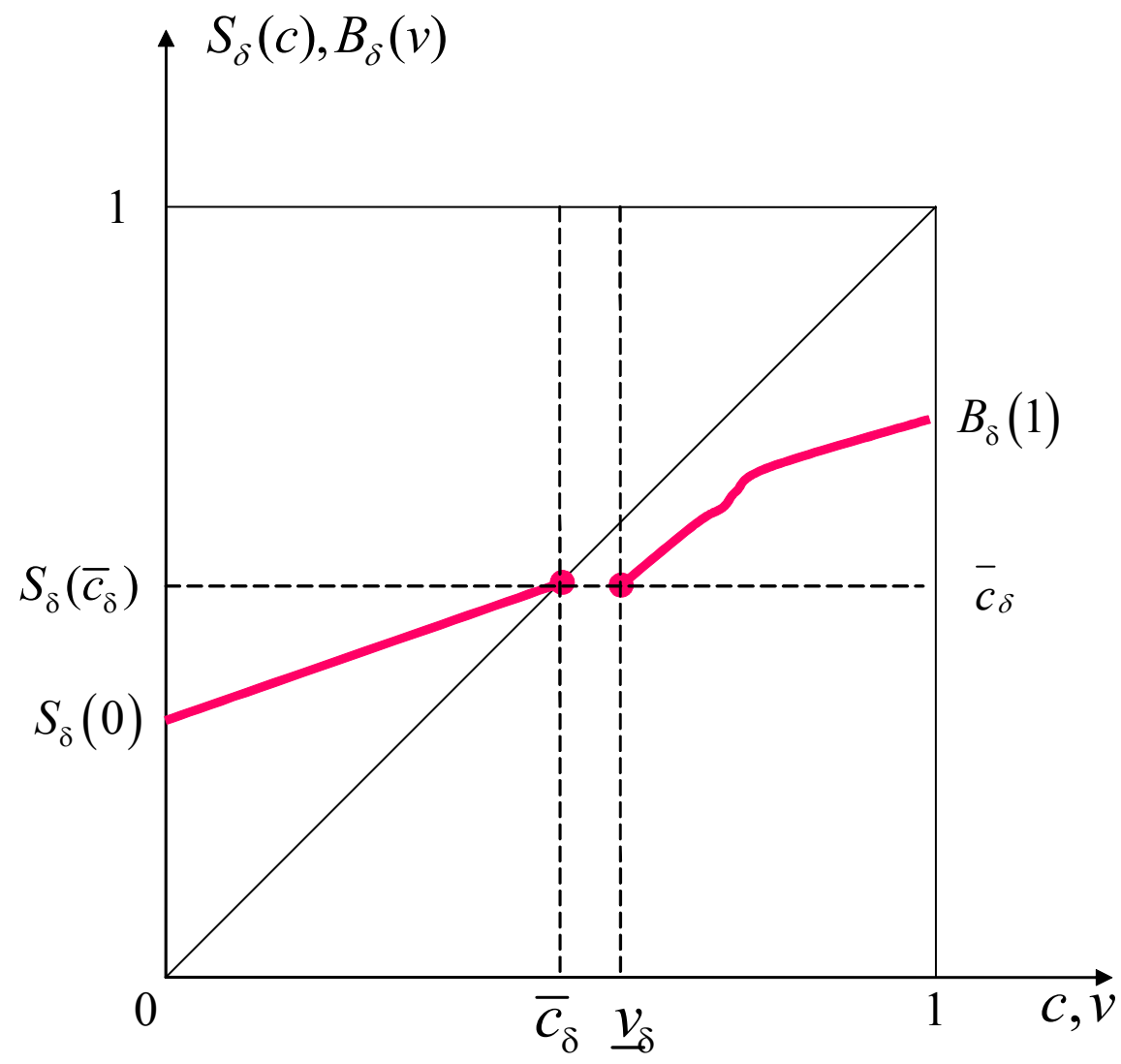

Figure 3: Strategies in a full-trade equilibrium 
The distribution $F_{B}$ is uniquely recoverable from $F_{B}^{*}$, the distribution of the maximal value. Equations (33) and (32)) imply that in the steady-state,

$$
\hat{\rho}_{B}(v)=e^{-\zeta}+\left(1-e^{-\zeta}\right) \frac{G_{B}(v)-G_{B}(\underline{v})}{1-G_{B}(\underline{v})} .
$$

On the other hand, direct computation shows that

$$
\begin{aligned}
\hat{\rho}_{B}(v) & =\sum_{k=0}^{\infty} e^{-\zeta} \frac{\zeta^{k}}{k !} F_{B}(v)^{k} \\
& =e^{-\zeta\left[1-F_{B}(v)\right]} \sum_{k=0}^{\infty} e^{-\zeta F_{B}(v)} \frac{\left[\zeta F_{B}(v)\right]^{k}}{k !} \\
& =e^{-\zeta\left[1-F_{B}(v)\right]}
\end{aligned}
$$

Equating the right-hand sides of (41) and (42) and solving, we obtain the unique steadystate distribution $F_{B}$ that corresponds to $\zeta$ and $\underline{v}$ in the characterizing equations' solution:

$$
F_{B}(v)=1+\frac{1}{\zeta} \log \left[e^{-\zeta}+\left(1-e^{-\zeta}\right) \frac{G_{B}(v)-G_{B}(\underline{v})}{1-G_{B}(\underline{v})}\right] .
$$

To complete the construction of the buyer's part of equilibrium, we must compute $T_{B}$, the steady-state mass of buyers. Mass balance of buyers implies

$$
T_{B} F_{B}^{\prime}(v) \hat{\rho}_{B}(v)=a \delta g_{B}(v) .
$$

Substitution of (41) and (43) into this and solving gives the formula:

$$
T_{B}=\zeta \frac{a \delta\left(1-G_{B}(\underline{v})\right)}{1-e^{-\zeta}}=\zeta \frac{a \delta \bar{G}_{B}(\underline{v})}{1-e^{-\zeta}} .
$$

A review of this construction shows that strategy $B$, the distribution $F_{B}^{*}$, and the mass $T_{B}$ depend only on the fundamentals $G_{B}, a, \delta$, and the solution $(\bar{c}, \underline{v}, \zeta)$ to the characterizing equations, but not the sellers' strategy $S$. This insulation of the buyers' optimal actions from the sellers' actions is the key insight behind this construction and overall proof design.

Turning to sellers, we already know the marginal participation type, $\bar{c}$, among sellers; it is is a component of $(\bar{c}, \underline{v}, \zeta)$. A seller trades in any period in which she is matched with at least one buyer; this probability is $\bar{\pi}_{0}=1-\pi_{0}=1-e^{-\zeta}$. Notice that it is independent of her type. Using formula (6), her discounted probability of trade is

$$
\hat{P}_{S}=\frac{1-e^{-\zeta}}{1-e^{-\beta \delta}+e^{-\beta \delta}\left(1-e^{-\zeta}\right)} .
$$

For active sellers - those with costs $c \leq \bar{c}$ - formula (14) gives their continuation values,

$$
\begin{aligned}
W_{S}(c) & =\int_{c}^{\bar{c}} \hat{P}_{S} d x \\
& =\hat{P}_{S}(\bar{c}-c),
\end{aligned}
$$


and formula (13) gives their equilibrium strategies,

$$
\begin{aligned}
S(c) & =c+e^{-\beta \delta} W_{S}(c) \\
& =c+e^{-\beta \delta} \hat{P}_{S}(\bar{c}-c) .
\end{aligned}
$$

Note that this is a linear function of $c$ with slope $1-e^{-\beta \delta} \hat{P}_{S}$. Sellers for whom $c>\bar{c}$ best-respond by not entering.

To construct the unique, steady state distribution of seller types, $F_{S}$, observe that the seller's best-response strategy $S$ is increasing on $[0, \bar{c}]$ and satisfies $S(\bar{c})=\bar{c}$. Since all active sellers trade with the same probability in any period, the distribution of their types in the market $F_{S}$ is just the distribution of the entering cohort conditional on $c \leq \bar{c}$ :

$$
F_{S}(c)=\frac{G_{S}(c)}{G_{S}(\bar{c})}
$$

To complete the construction of the seller's part of equilibrium, we must find the steadystate mass of active sellers $T_{S}$. Mass balance holds every period in a steady state; therefore $T_{S}\left(1-e^{-\zeta}\right)=\delta G_{S}(\bar{c})$ and, solving,

$$
T_{S}=\frac{\delta G_{S}(\bar{c})}{1-e^{-\zeta}} .
$$

To complete the proof, we need to check that, besides being mutual best-responses and inducing their own steady-state distributions $F_{B}$ and $F_{S}$, the strategies result in steady-state masses of buyers and sellers that have the required ratio $\zeta$ that was computed as a component of the solution to the characterizing equations. This is confirmed by dividing equation (45) by (48):

$$
\begin{aligned}
\frac{T_{B}}{T_{S}} & =\zeta \frac{a\left(1-G_{B}(\underline{v})\right)}{G_{S}(\bar{c})}=\zeta \frac{a \bar{G}_{B}(\underline{v})}{G_{S}(\bar{c})} \\
& =\zeta,
\end{aligned}
$$

where the last line follows from market clearing, equation (35).

We now turn to existence of equilibria for sufficiently small $\delta$. The second part of Proposition 13 implies that all we need to show is that, for sufficiently small $\delta$, a solution to the characterizing equations (35-37) exists. This is most easily done if we eliminate $\bar{c}$ from the system. Substitute

$$
\bar{c}=\underline{v}-\kappa \delta e^{\zeta}
$$

from equation (36) into equation (35) to obtain

$$
G_{S}\left(\underline{v}-\kappa \delta e^{\zeta}\right)-a\left(1-G_{B}(\underline{v})\right)=0 .
$$

Equation (35) can be re-written as

$$
\begin{aligned}
p & =\bar{c}+\frac{\kappa \delta}{1-e^{-\zeta}} \\
& =\underline{v}-\kappa \delta e^{\zeta}+\frac{\kappa \delta}{1-e^{-\zeta}}
\end{aligned}
$$


The new, two equation system in the three variables $(\underline{v}, \zeta, \delta)$ is then

$$
\begin{aligned}
G_{S}\left(\underline{v}-\kappa \delta e^{\zeta}\right)-a\left(1-G_{B}(\underline{v})\right) & =0 \\
p-\underline{v}+\kappa \delta \frac{e^{\zeta}-2}{1-e^{-\zeta}} & =0
\end{aligned}
$$

Recall that $p$ depends on $\underline{v}$ and $\zeta$ via (40), so it really is a system in three variables.

The method of proof we use has four steps. First - tediously - we substitute (40) into the system to eliminate $p$. Second, we extend the domain of system (51-52) from the economically meaningful set $D=(0,1) \times R \times(0, \infty)$ (i.e., $\delta$ is restricted to be positive) to the mathematically more useful set $D_{1}=(0,1) \times R \times(-1, \infty)$. Third, we prove that at $\delta=0$ the system has a unique solution $(\underline{v}, \zeta, \delta)=\left(p_{W}, \zeta_{0}, 0\right)$. Finally, we apply the implicit function theorem in a neighborhood of $\delta=0$ to establish that, for each $\delta$ in the neighborhood, a unique solution $\left(\underline{v}_{\delta}, \zeta_{\delta}, \delta\right)$ exists to the system. The complication in this step is showing that Jacobian of the system is non-zero at $\delta=0$. For each positive $\delta$ in the neighborhood, Proposition 13 implies that the solution $\left.\underline{v}_{\delta}, \zeta_{\delta}\right)$ characterizes a unique full-trade equilibrium.

Proposition 14 For all sufficiently small $\delta>0$. there exists a unique full-trade equilibrium $\left(\bar{c}_{\delta}, \underline{v}_{\delta}, \zeta_{\delta}\right)$.

Proof. By (40),

$$
p-\underline{v}=\frac{1}{1-G_{B}(\underline{v})} \int_{\underline{v}}^{1}(B(v)-\underline{v}) d G_{B}(v)
$$

and , by (39),

$$
\begin{aligned}
B(v)-\underline{v} & =v-\underline{v}-\int_{\underline{v}}^{v} \frac{\hat{P}_{B}(x)}{\hat{P}_{B}(v)} d x-\frac{\kappa \delta}{\hat{\rho}_{B}(v)} \\
& =\int_{\underline{v}}^{v}\left(1-\frac{\hat{P}_{B}(x)}{\hat{P}_{B}(v)}\right) d x-\frac{\kappa \delta}{\hat{\rho}_{B}(v)} .
\end{aligned}
$$

Algebra shows that

$$
1-\frac{\hat{P}_{B}(x)}{\hat{P}_{B}(v)}=\beta \delta \frac{1-e^{-\beta \delta}}{\beta \delta} \frac{\hat{\rho}_{B}(v)-\hat{\rho}_{B}(x)}{\hat{\rho}_{B}(v)\left(1-e^{-\beta \delta}+e^{-\beta \delta} \hat{\rho}_{B}(x)\right)} ;
$$

substituting this into (39) gives

$$
B(v)-\underline{v}=\delta\left(\beta \frac{1-e^{-\beta \delta}}{\beta \delta} \int_{\underline{v}}^{v} \frac{\hat{\rho}_{B}(v)-\hat{\rho}_{B}(x)}{\hat{\rho}_{B}(v)\left(1-e^{-\beta \delta}+e^{-\beta \delta} \hat{\rho}_{B}(x)\right)} d x-\frac{\kappa}{\hat{\rho}_{B}(v)}\right) .
$$

Inserting this expression for $B(v)-\underline{v}$ into (53) and substituting the resulting expression for $p-\underline{v}$ into (52) gives

$$
\delta L(\underline{v}, \zeta, \delta)=0,
$$


where

$$
\begin{aligned}
L(\underline{v}, \zeta, \delta)= & \frac{1}{1-G_{B}(\underline{v})} \int_{\underline{v}}^{1} \beta \frac{1-e^{-\beta \delta}}{\beta \delta} \int_{\underline{v}}^{v} \frac{\hat{\rho}_{B}(v)-\hat{\rho}_{B}(x)}{\hat{\rho}_{B}(v)\left(1-e^{-\beta \delta}+e^{-\beta \delta} \hat{\rho}_{B}(x)\right)} d x d G_{B}(v) \\
& -\frac{1}{1-G_{B}(\underline{v})} \int_{\underline{v}}^{1} \frac{\kappa}{\hat{\rho}_{B}(v)} d G_{B}(v) \\
& +\kappa \frac{e^{\zeta}-2}{1-e^{-\zeta}} .
\end{aligned}
$$

The second term of $L$ can be written as

$$
\begin{aligned}
& \frac{1}{1-G_{B}(\underline{v})} \int_{\underline{v}}^{1} \frac{\kappa}{\hat{\rho}_{B}(v)} d G_{B}(v) \\
= & \frac{\kappa}{1-G_{B}(\underline{v})} \int_{\underline{v}}^{1} \frac{1}{e^{-\zeta}+\left(1-e^{-\zeta}\right) \frac{G_{B}(v)-G_{B}(\underline{v})}{1-G_{B}(\underline{v})}} d G_{B}(v) \\
= & \frac{\kappa}{1-e^{-\zeta}} \log \frac{1}{e^{-\zeta}} \\
= & \frac{\kappa \zeta}{1-e^{-\zeta}}
\end{aligned}
$$

substituting this into (56) results in

$$
\begin{aligned}
L(\underline{v}, \zeta, \delta)= & \frac{1}{1-G_{B}(\underline{v})} \int_{\underline{v}}^{1} \beta \frac{1-e^{-\beta \delta}}{\beta \delta} \int_{\underline{v}}^{v} \frac{\hat{\rho}_{B}(v)-\hat{\rho}_{B}(x)}{\hat{\rho}_{B}(v)\left(1-e^{-\beta \delta}+e^{-\beta \delta} \hat{\rho}_{B}(x)\right)} d x d G_{B}(v) \\
& +\kappa \frac{e^{\zeta}-\zeta-2}{1-e^{-\zeta}}
\end{aligned}
$$

Simplifying further, $L(\underline{v}, \zeta, \delta)$ becomes:

$$
\begin{aligned}
L(\underline{v}, \zeta, \delta)= & \frac{1}{\left(1-G_{B}(\underline{v})\right)^{2}} \int_{\underline{v}}^{1} \beta \frac{1-e^{-\beta \delta}}{\beta \delta} \int_{\underline{v}}^{v} \frac{\left(1-e^{-\zeta}\right)\left(G_{B}(v)-G_{B}(x)\right)}{\hat{\rho}_{B}(v)\left(1-e^{-\beta \delta}+e^{-\beta \delta} \hat{\rho}_{B}(x)\right)} d x d G_{B}(v) \\
& +\kappa \frac{e^{\zeta}-\zeta-2}{1-e^{-\zeta}} .
\end{aligned}
$$

Given this work, the system (51-52) is, for $\delta>0$, equivalent to the system

$$
\begin{aligned}
G_{S}\left(\underline{v}_{\delta}-\kappa \delta e^{\zeta_{\delta}}\right)-a\left(1-G_{B}\left(\underline{v}_{\delta}\right)\right) & =0 \\
L\left(\underline{v}_{\delta}, \zeta_{\delta}, \delta\right) & =0
\end{aligned}
$$

where we have indexed $\underline{v}$ and $\zeta$ with $\delta$ to indicate that we solve this system for them as functions of $\delta$. Note that we have divided the second equation through by $\delta$; this is essential in order to ensure a non-zero Jacobian. Now extend the domain of the system from $D=(0,1) \times R \times(0, \infty)$ to $D_{1}=(0,1) \times R \times(-1, \infty)$; this domain includes the set $\left\{(\underline{v}, \zeta, \delta) \in D_{1}: \delta=0\right\}$. Recall formula (33) for $\hat{\rho}_{B}$ and observe that $\hat{\rho}_{B}(v), \hat{\rho}_{B}(x)$, 
and $\left(1-e^{-\beta \delta}\right) / \beta \delta$ are continuously differentiable functions of $\underline{v}, \zeta$ and $\delta$ on this larger domain. ${ }^{10}$ The function $L$, as a composition, is therefore continuously differentiable on $D_{1}$.

The first term in (57) is an increasing function of $\zeta$. This is because $1-e^{-\zeta}$ is increasing in $\zeta$ and $\hat{\rho}_{B}(\cdot)$ is decreasing in $\zeta$. The second term, $\left(e^{\zeta}-\zeta-2\right) /\left(1-e^{-\zeta}\right)$, is also increasing in $\zeta$, for

$$
\frac{d}{d \zeta} \frac{e^{\zeta}-\zeta-2}{1-e^{-\zeta}}=\frac{e^{\zeta}\left(\zeta+e^{2 \zeta}-3 e^{\zeta}+3\right)}{\left(1-e^{-\zeta}\right)^{2}}>0
$$

because $e^{2 \zeta}-3 e^{\zeta}+3=\left(e^{\zeta}-\frac{3}{2}\right)^{2}+3-\left(\frac{3}{2}\right)^{2}>0$. Therefore

$$
\frac{\partial L(\underline{v}, \zeta, \delta)}{\partial \zeta}>0
$$

i.e., $L$ is increasing in $\zeta$ for all values of $\underline{v}$ and $\delta$ in $D_{1}$.

This fact enables us to claim that a $\zeta_{0}$ exists such that $(\underline{v}, \zeta)=\left(p_{W}, \zeta_{0}\right)$ is the unique solution to system (58-59) at $\delta=0$. This is seen in two steps. First, when $\delta=0$ equation (58) reduces to $G_{S}\left(\underline{v}_{0}\right)-a\left(1-G_{B}\left(\underline{v}_{0}\right)\right)=0$, which is just equation (1) defining $p_{W}$, the Walrasian price. Therefore, at $\delta=0$ and irrespective of the value of $\zeta, p_{W}$ is the unique solution to (58), the system's first equation. Second, given that $\delta=0$ and $\underline{v}_{\delta}=p_{W}$, notice that $\lim _{\zeta \rightarrow 0} L\left(p_{W}, \zeta, 0\right)=-\infty$ and $\lim _{\zeta \rightarrow \infty} L\left(p_{W}, \zeta, 0\right)=\infty$ and recall that $L\left(p_{W}, \zeta, 0\right)$ is a continuous function increasing in $\zeta$. These facts together imply that a unique $\zeta_{0}$ exists solving $L\left(p_{W}, \zeta, 0\right)=0$. Thus, as claimed, $(\underline{v}, \zeta)=\left(p_{W}, \zeta_{0}\right)$ is the unique solution to the system at $\delta=0$.

The Jacobian $J$ of the system $(58-59)$ at $(\underline{v}, \zeta, \delta)=\left(p_{W}, \zeta_{0}, 0\right)$ is not zero:

$$
\begin{aligned}
J & =\left|\begin{array}{cc}
g_{S}\left(p_{W}\right)+a g_{B}\left(p_{W}\right) & 0 \\
* & \frac{\partial L\left(p_{W}, \zeta_{0}, 0\right)}{\partial \zeta}
\end{array}\right| \\
& =\left(g_{S}\left(p_{W}\right)+a g_{B}\left(p_{W}\right)\right) \frac{\partial L\left(p_{W}, \zeta_{0}, 0\right)}{\partial \zeta}>0
\end{aligned}
$$

where the last line follows from inequality (60). Consequently, the implicit function theorem applies so for all small enough $\delta>0$ a unique solution $\left(\underline{v}_{\delta}, \zeta_{\delta}\right)$ exists. Given that for positive $\delta$ a one-to-one map exists between $\left(\underline{v}_{\delta}, \zeta_{\delta}\right)$ pairs and full trade equilibria, this proves that there is a unique full-trade equilibrium for all sufficiently small $\delta>0$. This proves Proposition 14. It also proves Theorem 2, which states that for sufficiently small $\delta$ an equilibrium (not necessarily full trade) exists. Theorem 2 makes no mention of uniqueness because, for a particular value of $\delta$, the existence of a unique full trade equilibrium does not rule out another equilibrium that is not full trade.

\footnotetext{
${ }^{10}$ The expression $\left(1-e^{-\beta \delta}\right) / \beta \delta$ is indeterminate at $\delta=0$, but selecting $\lim _{\delta \rightarrow 0}\left(1-e^{-\beta \delta}\right) / \beta \delta$ to be its value at that point makes it continuous and differentiable over all of $D_{1}$.
} 


\section{Conclusions}

In this paper we have shown that convergence to the competitive price and allocation can be achieved with a decentralized matching and bargaining market in which all traders have private information about their values/costs. The significance of this contribution is that it directly addresses a critical shortcoming in each of two literatures it combines. Existing matching and bargaining models that demonstrate robust convergence ignore the ubiquity of incomplete information. Existing double auction models robustly demonstrate convergence in the presence of incomplete information, but ignore the equally ubiquitous future opportunities for trade that exist in almost all real markets. Our model and results cure both these shortcomings.

Of the many open questions that remain we briefly discuss three of the most important. First, no constrained optimal benchmark has been derived for the dynamic matching and bargaining model with incomplete information. Presumably mechanism design techniques can be used to establish such a benchmark. ${ }^{11}$ Then it would be possible to compare the realized efficiency of models such as ours that have specific matching and bargaining protocols against the efficiency of the constrained optimal mechanism.

Second, while our model provides a set of natural sufficient conditions for convergence, an appropriate long term goal is to characterize fully the conditions that are both necessary and sufficient for convergence. The paper of Wolinsky (1988) suggests that a homogeneous product is necessary. Similarly, the papers of Serrano (2002) and DeFraja and Sákovics (2002) suggests that global market clearing operating through endogenous distributions of active traders is necessary in some form. Additionally Serrano's paper suggests that admitting a continuum of bid values may be necessary.

Third, we assume both an independent private value environment and that sellers have unit supply and buyers have unit demand. Recent papers by Fudenberg, Mobius, and Szeidl (2003), Cripps and Swinkels (2003), and Reny and Perry (2003) on the static double auction have relaxed these assumptions. Specifically, Fudenberg, Mobius, and Szeidl show existence and a rate of convergence to truth-telling in a model with correlated private costs/values. Cripps and Swinkels, using a more general model of correlated private values, dispense with the unit supply/unit demand assumption and show that the relative inefficiency of the static double auction is $O\left(\frac{1}{n^{2-\varepsilon}}\right)$ where $\varepsilon$ is an arbitrarily small constant. Reny and Perry allow traders' costs/values to have a common value component and their private signals to be affiliated. They show that, if the market is large enough, an equilibrium exists, is almost ex post efficient, and almost fully aggregates the traders' private information, i.e., the double auction equilibrium approaches the unique, fully revealing rational expectations equilibrium that exists in the limit. Obviously generalizing our model in these same directions is important.

If these and other questions can be answered adequately in future work, then this theory may become useful in designing and regulating decentralized markets with incomplete information in much the same way auction theory has become useful in designing auctions. The ubiquity of the Internet with its capability for facilitating matches and

\footnotetext{
${ }^{11}$ Satterthwaite and Williams (2002) have done this for static double auctions.
} 
reducing period length makes pursuit of this end attractive.

\section{References}

[1] Butters, G. R. Circa 1979. Equilibrium price distributions in a random meetings market. Working paper.

[2] Cripps, M. and J. Swinkels. 2003. Efficiency in Large Double Auctions. Working paper, October.

[3] De Fraja, G. and J. Sákovics 2001. Walras retrouvé: Decentralized trading mechanisms and the competitive price. J. of Political Economy 109 (August): 842-63.

[4] Fudenberg, D., M. M. Mobius, and A. Szeidl. 2003. Existence of equilibrium in large double auctions. Working paper, April.

[5] Gale, D. 1986. Bargaining and competition Part I: Characterization. Econometrica 54 (July): 785-806.

[6] Gale, D. 1987. Limit theorems for markets with sequential bargaining. J. of Economic Theory 43 (October): 20-54.

[7] Gale, D. 2000. Strategic Foundations of General Equilibrium: Dynamic Matching and Bargaining Games. Cambridge: Cambridge University Press.

[8] Milgrom, P. 2004. Putting Auction Theory to Work. Cambridge: University Press.

[9] Milgrom, P. and I. Segal. 2002. Envelope theorems for arbitrary choice sets. Econometrica 70 (March): 583-601.

[10] Mortensen, D. and R. Wright. 2002. Competitive pricing and efficiency in search equilibrium. International Economic Review 43 (February): 1-20.

[11] Osborne, M. and A. Rubinstein. 1990. Bargaining and Markets. San Diego: Academic Press.

[12] Peters, M. and S. Severinov. 2002. Internet Auctions with Many Traders. Working Paper, University of British Columbia.

[13] Reny, P. J. and M. Perry. 2003. Toward a strategic foundation of rational expectations equilibrium. Working paper, June.

[14] Rubinstein, A. and A. Wolinsky. 1985. Equilibrium in a market with sequential bargaining. Econometrica 53 (September): 1133-50.

[15] Rubinstein, A. and A. Wolinsky. 1990. Decentralized trading, strategic behavior, and the Walrasian outcome. Review of Economic Studies 57 (January): 63-78. 
[16] Rust, J. and G. Hall. 2002. Middlemen versus market makers: A theory of competitive exchange. J. of Political Economy 111: 353-403.

[17] Rustichini, A., M. Satterthwaite, and S. Williams. 1994. Convergence to efficiency in a simple market with incomplete information. J. of Economic Theory 62 (September): 1041-63.

[18] Satterthwaite, M. and S. Williams. 1989. The rate of convergence to efficiency in the buyer's bid double auction as the market becomes large. Review of Economic Studies 56: 477-98.

[19] Satterthwaite, M. and S. Williams, 2002, The Optimality of a Simple Market Mechanism, Econometrica 70: 1841-63.

[20] Satterthwaite, M and A. Shneyerov, 2003, "Convergence of a Dynamic Matching and Bargaining Market with Two-sided Incomplete Information to Perfect Competition". CMS-EMS Working paper 1384, Northwestern University.

[21] Serrano, R. 2002. Decentralized information and the Walrasian outcome: A pairwise meetings market with private values. J. of Mathematical Economics 38: 65-89.

[22] Shiryaev, A. 1995. Probability. Graduate texts in Mathematics No. 95, 2nd edition. New York: Springer-Verlag.

[23] Spulber, D. 1999. Market Microstructure: Intermediaries and the Theory of the Firm. Cambridge: Cambridge University Press.

[24] Wolinsky, A. 1988. Dynamic markets with competitive bidding. Review of Economic Studies 55 (January): 71-84. 